\title{
Connectedness of Efficient Solutions in Multiple Objective Combinatorial Optimization
}

\author{
Jochen Gorski* Kathrin Klamroth* $\quad$ Stefan Ruzika ${ }^{\dagger}$
}

\begin{abstract}
Connectedness of efficient solutions is a powerful property in multiple objective combinatorial optimization since it allows the construction of the complete efficient set using neighborhood search techniques. In this paper we show that, however, most of the classical multiple objective combinatorial optimization problems do not possess the connectedness property in general, including, among others, knapsack problems (and even several special cases of knapsack problems) and linear assignment problems. We also extend already known non-connectedness results for several optimization problems on graphs like shortest path, spanning tree and minimum cost flow problems. Different concepts of connectedness are discussed in a formal setting, and numerical tests are performed for different variants of the knapsack problem to analyze the likelihood with which non-connected adjacency graphs occur in randomly generated problem instances.
\end{abstract}

Keywords: Multiple objective combinatorial optimization; MOCO; connectedness; adjacency; neighborhood search

\section{Introduction and problem formulation}

Multiple objective combinatorial optimization (MOCO) has become a quickly growing field in multiple objective optimization, and has recently attracted the attention of researchers both from the fields of multiple objective optimization and from single objective

\footnotetext{
*Institute of Applied Mathematics, University of Erlangen-Nuremberg

${ }^{\dagger}$ Department of Mathematics, University of Kaiserslautern. His research was partially supported by Deutsche Forschungsgemeinschaft (DFG) grant HA 1737/7 "Algorithmik großer und komplexer Netzwerke".
} 
integer programming (see, for example, Ehrgott and Gandibleux (2000) for a recent review). Typical examples of MOCO problems include multiple objective knapsack problems (MOKP) with applications, among others, in capital budgeting, multiple objective assignment problems (MOAP), the multiple objective traveling salesman problem (MTSP), and other network problems like multiple objective minimum spanning tree (MOST), shortest path (MOSP), and minimum cost flow (MOMC) problems.

Structural properties of the efficient set of MOCO problems play a crucial role for the development of efficient solution methods. A central question relates to the connectedness of the efficient set with respect to combinatorially or topologically motivated neighborhood structures. A positive answer to this question would provide a theoretical justification for the application of fast neighborhood search techniques, not only for multiple objective but also for appropriate formulations of single objective problems.

Formally, a general MOCO problem can be stated as

$$
\min f(x)=\left(f_{1}(x), \ldots, f_{p}(x)\right)^{T}
$$

s.t. $x \in X$,

where the decision space $X$ is a given discrete feasible set with some additional combinatorial structure. The vector-valued objective function $f: X \longrightarrow \mathbb{Z}^{p}$ maps the feasible solutions into the objective space. $Y:=f(X)$ denotes the image of the feasible set in the objective space.

We use the Pareto concept of optimality for MOCO problems which is based on the componentwise ordering of $\mathbb{Z}^{p}$ defined for any pair $y^{1}, y^{2} \in \mathbb{Z}^{p}$ by

$$
\begin{aligned}
& y^{1} \leqq y^{2} \quad \Leftrightarrow \quad y_{k}^{1} \leq y_{k}^{2}, \quad k=1, \ldots, p \\
& y^{1} \leq y^{2} \quad \Leftrightarrow \quad y_{k}^{1} \leq y_{k}^{2}, \quad k=1, \ldots, p \quad \text { and } \quad y^{1} \neq y^{2} \\
& y^{1}<y^{2} \quad \Leftrightarrow \quad y_{k}^{1}<y_{k}^{2}, \quad k=1, \ldots, p .
\end{aligned}
$$

A point $y^{2} \in \mathbb{Z}^{p}$ is called dominated by $y^{1} \in \mathbb{Z}^{p}$ if $y^{1} \leq y^{2}$. According to the Pareto concept of optimality, the efficient set $X_{E}$ and the weakly efficient set $X_{w E}$ are defined as

$$
\begin{aligned}
X_{E} & :=\{x \in X: \text { there exists no } \bar{x} \in X \text { with } f(\bar{x}) \leq f(x)\} \\
X_{w E} & :=\{x \in X: \text { there exists no } \bar{x} \in X \text { with } f(\bar{x})<f(x)\} .
\end{aligned}
$$

The images $Y_{N}:=f\left(X_{E}\right)$ and $Y_{w N}:=f\left(X_{w E}\right)$ of these sets under the vector-valued mapping $f$ are called the nondominated set and the weakly nondominated set, respectively.

In continuous optimization connectedness is defined in a topological sense. A set $S$ is called topologically connected if there does not exist nonempty open sets $S_{1}$ and $S_{2}$ such that $S \subseteq S_{1} \cup S_{2}$ and $S_{1} \cap S_{2}=\emptyset$. For multiple objective linear programming (MOLP) 
problems the efficient set and the nondominated set are topologically connected as shown by Naccache (1978) and Warburton (1983), respectively. This definition is not directly applicable in combinatorial optimization due to the discrete structure of the efficient set. Instead of the topological connectedness, a graph theoretical definition can be used for MOCO problems as described, for example, in Ehrgott and Klamroth (1997) and Paquete and Stützle (2006).

Definition 1.1 For a given MOCO problem the adjacency graph of efficient solutions $\mathcal{G}=(\mathcal{V}, \mathcal{A})$ of the $M O C O$ problem is defined as follows: $\mathcal{V}$ consists of all efficient solutions of the given MOCO problem. An (undirected) edge is introduced between all pairs of vertices which are adjacent with respect to the considered definition of adjacency for the given MOCO problem. These edges form the set $\mathcal{A}$.

The connectedness of $X_{E}$ is now defined via the connectedness of an undirected graph. We recall that an undirected graph $\mathcal{G}$ is said to be connected if every pair of vertices is connected by a path.

Definition 1.2 The set $X_{E}$ of all efficient solutions of a given MOCO problem is said to be connected if its corresponding adjacency graph $\mathcal{G}$ is connected.

Since for MOCO problems the adjacency of two efficient solutions $x$ and $x^{\prime}$ can usually be expressed by an application of some so-called elementary move (i.e., $x$ can be obtained from $x^{\prime}$ by applying exactly one move), a neighborhood concept is introduced to the problem. An efficient solution $x^{\prime}$ is contained in the $k$-neighborhood of an efficient solution $x$ if $x^{\prime}$ is reachable from $x$ by applying at most $k$ elementary moves. The minimum number of elementary moves needed to get from $x$ to $x^{\prime}$ is called the distance between these two solutions. Using this concept, the definition of the adjacency graph can be further extended.

Definition 1.3 The weighted adjacency graph $\mathcal{G}^{\prime}=\left(\mathcal{V}^{\prime}, \mathcal{A}^{\prime}\right)$ of efficient solutions is defined as follows: $\mathcal{G}^{\prime}$ is a complete and undirected graph. Its set of vertices $\mathcal{V}^{\prime}$ consists of all efficient solutions of the given MOCO problem. The weight $w_{i j}$ of an arc between to vertices $v_{i}, v_{j} \in \mathcal{V}^{\prime}$ is given by the distance between these two vertices with respect to the considered neighborhood.

For each $k \in \mathbb{N}$ a subgraph $\mathcal{G}_{k}^{\prime}$ can be extracted from $\mathcal{G}^{\prime}$ that contains all the vertices of $\mathcal{G}^{\prime}$ but only those arcs which have a weight less or equal to $k$. Since $\mathcal{G}=\mathcal{G}_{1}^{\prime}, X_{E}$ is connected if and only if $\mathcal{G}_{1}^{\prime}$ is connected. If $X_{E}$ is not connected, the graph $\mathcal{G}_{1}^{\prime}$ decomposes into at least two connected subgraphs which build a partition of $\mathcal{G}_{1}^{\prime}$. More generally we define: 
Definition 1.4 A component or a cluster of efficient solutions at distance $k$ is a maximal connected subgraph of $\mathcal{G}_{k}^{\prime}$.

If $\mathcal{G}_{k}^{\prime}$ is a connected graph, there exists exactly one component which is equal to $\mathcal{G}_{k}^{\prime}$. Otherwise, the set of all components build a partition of $\mathcal{G}_{k}^{\prime}$.

\section{Literature review}

The literature on the connectedness of the set of efficient solutions in multiple objective optimization is scarce. The first publications appeared in the seventies together with the development of the multiple objective simplex method. In his fundamental work, Isermann (1977) showed that the set of basic feasible and fundamental solutions of MOLP problems are connected and, thus, established the correctness of multiple objective simplex methods. Two solutions of an MOLP problem are said to be adjacent in the sense of Isermann (1977) if they have $m-1$ basic variables in common. Naccache (1978) established connectivity for more general problems with closed, convex and $K$-compact outcome spaces where $K$ is a closed, convex and pointed cone. Helbig (1990) generalized this to locally convex spaces.

Lately, research on the connectedness of efficient solutions of MOCO problems was coined by assertions and falsifications. Martins (1984) claimed that there always exists a sequence of adjacent efficient paths connecting two arbitrary efficient paths for MOSP.

However, Ehrgott and Klamroth (1997) demonstrated the incorrectness of the connectedness conjecture for MOSP and MOST problems by a counterexample and, thus, disproved Martins (1984). Ehrgott and Klamroth (1997) showed that any graph can be extended in such a way that the adjacency graph (of MOSP and MOST) for the problem on the extended graph is not connected. They conjectured that in practice, it is rather unlikely that the adjacency graph of a specific MOST problem is not connected. However, their numerical tests included only 50 randomly generated graphs with a rather small number of nodes.

In Przybylski et al. (2006), the example of Ehrgott and Klamroth (1997) was used to show the incorrectness of the algorithm of Sedeño-Noda and González-Martín (2001). The latter tried to find all efficient flows of a biobjective integer flow problem by a method based on simplex pivots.

O'Sullivan and Walker (2004) proposed two algorithms for the equally-weighted biobjective knapsack problem the success of which depends on "unproven characteristics of efficient knapsacks" - the connectedness of the set of efficient solutions for this problem.

In da Silva et al. (2004), the geometrical configuration of the nondominated set for three different models of the biobjective $\{0,1\}$-knapsack problem was discussed. Under 
a cardinality constraint and the supplementary assumption that the sum of each pair of the objective coefficients is constant, it was shown that the set of all efficient solutions is connected. In this case, the nondominated set consists of a line segment with slope -1 . An LP-based approach is used to define adjacency of two efficient solutions.

Gorski (2004) recognized that the definition of adjacency is not canonical. One could think of structural, problem-dependent definitions or of LP-based, problem independent definitions. Based on ideas mentioned in Ehrgott and Klamroth (1997), he aimed at a formal definition of adjacency.

The numerical study of Paquete et al. (2004) investigates the number of clusters of near efficient solutions obtained with some local search algorithms for the MTSP. In Paquete and Stützle (2006) statistics on the clusters of near efficient solutions for the biobjective travelling salesman problem and the biobjective quadratic assignment problem are reported. A stochastic local search method was employed to retrieve the near optimal solutions. It should be pointed out that neither the solutions obtained are guaranteed to be efficient, nor that all efficient solutions are found by the local search method. Thus, the focus of this study is on the performance assessment of local search for the two MOCO problems mentioned.

Some comments on the connectedness of efficient solutions for biobjective multimodal assignment problems are also contained, but not further persued, in Pedersen (2006).

\section{Contribution of this work}

The remainder of this article is organized as follows. In Section 2, we discuss different definitions of adjacency of feasible solutions of a MOCO problem. On one hand, adjacency may be defined based on appropriate IP-formulations of a given problem and using the natural neighborhood of basic feasible solutions of linear programming. For many concrete problems, however, it appears to be more convenient to consider a combinatorial neighborhood. In Section 3 we discuss and extend existing results for the MOSP and the MOST problem and present new connectedness results for other major classes of MOCO problems like the MOKP, the MOAP and the MTSP, among others. We report numerical tests on adjacency of efficient solutions for the binary MOKP with bounded cardinalities and the binary multiple choice MOKP in Section 4. Finally, we conclude the paper in Section 5 with current and future research ideas.

\section{Categorizing different concepts of adjacency}

We distinguish between two essentially different concepts of adjacency of efficient solutions: 
- The adjacency of two efficient solutions is defined via the adjacency of basic feasible solutions of an appropriate model of the MOCO problem as a multiple objective integer linear programming (MILP) problem, and its LP relaxation.

- The definition of adjacency is adapted to the special combinatorial structure of the given MOCO problem.

While the latter concept has received some attention in the recent literature, for example, in the context of neighborhood search algorithms (see, for example, Paquete and Stützle, 2006), the former has only been used so far for special types of MOCO problems (cf. Ehrgott and Klamroth (1997), for the MOSP and the MOST and da Silva et al. (2004) for $\{0,1\}$-knapsack problems). Subsequently, we formalize these two concepts of adjacency.

\subsection{MILP-based definition of adjacency and appropriate MILP models}

For the MILP-based definition, we assume that the MOCO problem can be formulated as a combinatorial optimization problem with weighted-sum type objective functions as specified in Definition 2.1 below.

Definition 2.1 Let $E:=\left\{e_{1}, \ldots, e_{n}\right\}$ be a nonempty finite set, let $c: E \rightarrow \mathbb{Z}^{p}, p>1$, be an integer-valued weighting function on the elements of $E$, and let $\mathcal{Z} \subseteq \mathcal{P}(E)$ be a subset of the powerset of $E$. A multiple objective combinatorial sum problem $(E, \mathcal{Z}, c)$ is a problem of the form

$$
\min \left\{\sum_{e \in Z} c(e): Z \in \mathcal{Z}\right\} .
$$

As described in Ehrgott (2005), every feasible solution $Z \in \mathcal{Z}$ of a MOCO problem (1) can be identified with a binary vector $x \in\{0,1\}^{n}$ by setting

$$
x_{i}:= \begin{cases}1 & \text { if } e_{i} \in Z \\ 0 & \text { otherwise. }\end{cases}
$$

The weight $\sum_{e \in Z} c(e)$ of a solution $Z$ and of its binary representation $x$ can be computed as $C x$ using an appropriate objective matrix $C \in \mathbb{Z}^{p \times n}$ with components $c_{i j}:=c_{i}\left(e_{j}\right)$ for $i=1, \ldots, p$ and $j=1 \ldots, n$.

Theorem 2.1 Every multiple objective combinatorial sum problem (1) can be modeled as an MILP problem of the form

$$
\min \left\{C x: A x \leqq b, x \in\{0,1\}^{n}\right\},
$$


using variables $x$ as defined in (2) and with an appropriate constraint matrix $A \in \mathbb{Z}^{m \times n}$ and right-hand-side vector $b \in \mathbb{Z}^{m}$, such that there is a one-to-one correspondence between all feasible (and particulary all efficient) solutions of the two problems.

Proof: See Ehrgott (2005) and Gorski (2004).

We refer to a formulation of a MOCO problem according to Theorem 2.1 as a canonical MILP formulation of the MOCO problem. Note that a canonical MILP formulation is in general not unique. For the sake of simplicity we assume in the following that a canonical MILP formulation is given. This is, however, not a necessary assumption for our analysis. Namely, instead of a canonical MILP formulation we may consider any MILP formulation of the problem that satisfies the conditions of Definition 2.3 below, i.e., there is a one-toone correspondence between the feasible solutions of the MOCO problem and the extreme points of the LP relaxation of the MILP problem.

Denote by $U:=\left\{x \in\{0,1\}^{n}: A x \leqq b\right\}, P:=\left\{x \in[0,1]^{n}: A x \leqq b\right\}$ and $P^{*}:=\{x \in$ $\left.\mathbb{R}^{n}: x \in \operatorname{conv}(U)\right\}$ the feasible set of a canonical MILP formulation, the feasible set of the LP relaxation of the MILP problem, and the convex hull of all feasible solutions of the MILP problem, respectively.

Definition 2.2 If the polytope $P$ of the LP relaxation of a canonical MILP formulation coincides with the polytope $P^{*}$, we say that $\min \left\{C x: A x \leqq b, x \in\{0,1\}^{n}\right\}$ is an exact MILP formulation of the given MOCO problem.

In order to use an LP-based definition of adjacency, a one-to-one correspondence between feasible solutions of the MOCO problem and basic feasible solutions of the LP relaxation of the corresponding MILP formulation is indispensable. We therefore restrict our analysis to exact MILP formulations of a given MOCO problem. Note that otherwise there may exist basic feasible solutions of the LP relaxation of the (non-exact) MILP formulation which are not integer and which do not correspond to feasible solutions of the MOCO problem. On the other hand, every feasible solution of the MOCO problem must correspond not only to a feasible solution of its (exact) MILP formulation, but to a basic feasible (or extreme point) solution of the LP relaxation of the MILP problem.

Definition 2.3 An MILP formulation of a given MOCO problem is called appropriate if it satisfies the following two conditions:

- The MILP formulation is exact, i.e., $P=P^{*}$.

- Every feasible solution of the MILP problem is an extreme point of $P^{*}$. 
Polyhedral theory can be used to show that for every MOCO problem at least one appropriate MILP formulation exists.

Lemma 2.1 There exists at least one appropriate MILP formulation for every MOCO problem $(E, \mathcal{Z}, c)$.

Proof: Suppose that an arbitrary MOCO problem $(E, \mathcal{Z}, c)$ is given, let $U$ denote the set of all feasible binary vectors for the canonical formulation of the MOCO problem (cf. Theorem 2.1), and let $P^{*}$ denote the convex hull of $U$. Then an exact formulation of the problem is given by

$$
\min \{C x: x \in U\} \text {. }
$$

Since all vectors $x \in U$ are binary, they are essential for the generation of the convex hull $P^{*}$ of $U$. Hence, problem (3) is equivalent to

$$
\min \left\{C x: x \in P^{*}, x \in\{0,1\}^{n}\right\},
$$

and every feasible solution of this problem is an extreme point of $P^{*}$. Moreover, since all feasible vectors $x \in U$ are essential for the generation of $P^{*}$, there exists a description of $P^{*}$ by means of a finite set of linear inequalities of the form $P^{*}=\left\{x \in \mathbb{R}^{n}: \tilde{A} x \leqq \tilde{b}\right\}$ with appropriate rational $\tilde{A} \in \mathbb{Z}^{\tilde{m} \times n}$ and $b \in \mathbb{Z}^{\tilde{m}}$ (see, for example, Nemhauser and Wolsey, 1999), yielding an appropriate MILP formulation for problem (4) and hence for the MOCO problem.

Note that the polytope $P^{*}$ of an appropriate MILP formulation of MOCO problem does not contain any integer points in its interior nor in the interior of any of its faces.

The following two properties can be derived for an appropriate MILP formulation of a MOCO problem which will be used later for the LP-based definition of adjacency.

Lemma 2.2 If an MILP formulation of a MOCO problem is appropriate, then its LP relaxation, after transformation into standard form, has the following two properties:

(M1) Every basic feasible solution corresponds to a feasible solution of the MOCO problem.

(M2) For every feasible solution of the MOCO problem there exists at least one basis such that the solution of the MOCO problem is equal to the corresponding basic feasible solution of the above LP relaxation of the MILP problem in standard form.

Proof: Follows immediately from the analysis above and from polyhedral theory. 
Lemma 2.3 Suppose that a MOCO problem is given by an (arbitrary binary) MILP formulation. If the LP relaxation of the MILP problem, after transformation into standard form, satisfies (M1) and (M2), then the MILP problem is an appropriate formulation of the MOCO problem.

Proof: Let the MILP formulation of the MOCO problem be given as $\min \{C x: A x \leqq$ $\left.b, x \in\{0,1\}^{n}\right\}$ and let $U, P$ and $P^{*}$ be defined as above. Furthermore, let $U_{p}$ denote the finite set of all extreme points of $P$. Since we assumed that the MOCO problem is finite and $E \neq \emptyset, P$ is bounded and hence $P=\operatorname{conv}\left\{u_{p} \in U_{p}\right\}$.

We first show that $P=P^{*}$. By construction we have that $P^{*} \subseteq P$. To show that also $P \subseteq P^{*}$ suppose that, to the contrary, there exists an extreme point $x^{0} \in U_{p} \subseteq P$ that is not contained in $P^{*}$. Since $x^{0} \in P, x^{0}$ satisfies $A x^{0} \leqq b$ and $x^{0} \in[0,1]^{n}$. Furthermore, since $x^{0}$ is an extreme point of $P$ there exists a basic feasible solution of the LP relaxation of the MILP problem (after transformation into standard form) corresponding to $x^{0}$. Hence, $x^{0} \in\{0,1\}^{n}$ by (M1) and therefore $x^{0} \in U$, contradicting the assumption that $x^{0} \notin P^{*}$.

Since (M2) and Theorem 2.1 imply that there is a one-to-one correspondence between feasible solutions $u$ of the MILP problem and feasible solution $Z \in \mathcal{Z}$ of the MOCO problem, the MILP problem is indeed an appropriate formulation of the MOCO problem.

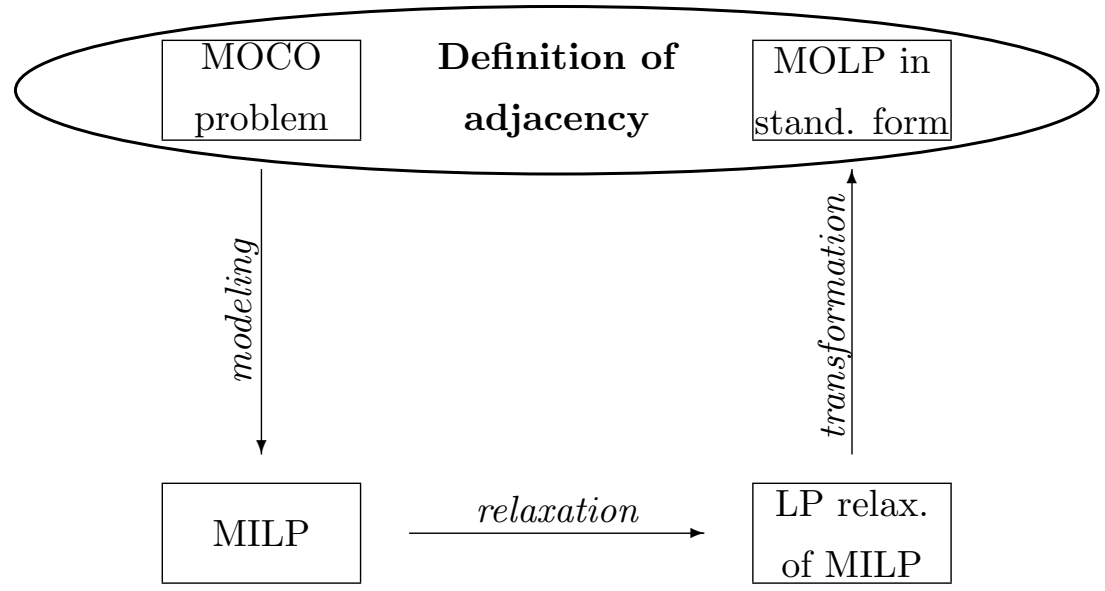

Figure 1: Definition of adjacency via an appropriate MILP formulation.

Since properties (M1) and (M2) characterize appropriate MILP formulations of MOCO problems, they can be used for the definition of an LP-based concept of adjacency for these problems. Figure 1 illustrates this idea. In this context, two bases of an LP are called adjacent if they can be obtained from each other by one single pivot operation. 
Definition 2.4 Let an appropriate MILP formulation of a MOCO problem be given. Two feasible solutions $x^{1}$ and $x^{2}$ of the MOCO problem are called adjacent with respect to the given MILP formulation if there exist two adjacent bases of the LP relaxation of the MILP problem (after transformation into standard form) corresponding to $x^{1}$ and $x^{2}$, respectively.

Since by MOLP theory all bases which represent efficient solutions of the LP relaxation of the MILP problem in standard form are connected (see, for example, Ehrgott, 2005), the resulting adjacency graph always contains a connected subgraph representing these solutions. Note that these solutions are always supported efficient solutions of the MOCO problem.

The above definition of adjacency (and hence the resulting adjacency graph) depends on the chosen appropriate MILP formulation of the given MOCO problem, which is in general not unique. If different appropriate MILP formulations are used to model the same MOCO problem, we can expect different results concerning the connectedness of efficient solutions of the problem. In this context, Definitions 1.1 and 1.2 must always be understood with respect to the chosen appropriate MILP formulation of a MOCO problem.

Note also that, using the above definitions of adjacency and connectedness, polyhedral theory implies that the set of optimal solutions of a single objective combinatorial optimization problem is always connected (or even unique). Therefore, the question whether the corresponding multiple objective optimization problems have a connected adjacency graph is in general non-trivial.

The following well-known fact from polyhedral theory shows that the last step in Figure 1, i.e., the transformation of the LP relaxation of the MILP problem into standard form, can as well be omitted in the definition of adjacency (Definition 2.4) since the considered MILP problems are always bounded problems.

Proposition 2.1 Let $P=\left\{x \in[0,1]^{n}: A x \leqq b\right\}$ be the feasible set of an LP and let $P_{\mathrm{st}}$ denote the polyhedron obtained from $P$ after transformation into standard from. Then two extreme points of $P$ are connected by an edge in $P$ if and only if the corresponding extreme points of $P_{\mathrm{st}}$ are connected by an edge in $P_{\mathrm{st}}$.

\subsection{Combinatorial definitions of adjacency}

Combinatorial definitions of adjacency are usually based on simple operations that transform one feasible solution of a specific problem class into another, "adjacent" feasible solution. We call such operations (elementary) moves. An elementary move is called efficient if it leads from one efficient solution of the problem to another efficient solution. 
Two efficient solutions are called adjacent if one can be obtained from the other by one efficient move.

Examples for elementary moves for specific problem classes are the insertion and deletion of arcs in a spanning tree, the modification of a matching along an alternating cycle, or simply the swap of two bits in a binary solution vector. In single objective optimization such elementary moves are frequently used in exact algorithms (e.g., the negative dicycle algorithm for the minimum cost flow problem) as well as in heuristic algorithms (e.g., the two-exchange heuristic for the TSP). Note that for specific problem classes, a combinatorial definition of adjacency may in fact coincide with an MILP-based definition of adjacency as discussed in Section 2.1.

While for the MILP-based definition of adjacency the set of optimal solutions of the single objective problem corresponding to a given MOCO problem is always connected in the sense of Definition 1.1 (cf. Section 2.1), this is not necessarily true for combinatorial definitions of adjacency. We call an elementary move for a given problem class canonical if the set of optimal solutions of the corresponding single objective problem is connected for all problem instances. Although non-canonical moves immediately imply non-connectedness results also in the multiple objective case, such extensions may be used for the development of heuristic methods based on neighborhood search (see, for example, Paquete and Stützle, 2006).

For some classes of combinatorial problems, an elementary move corresponds to a move from one extreme point to another adjacent extreme point along an edge of the polytope which is obtained by the LP relaxation of an MILP formulation of the given combinatorial problem (cf. Definition 2.4). If the given MILP formulation is appropriate in the sense of Definition 2.3, the corresponding elementary move is always canonical.

Proposition 2.2 Let a move-operation introduce a combinatorial definition of adjacency for a class of MOCO problems for which also an appropriate MILP formulation exists, and let $P$ denote the polytope of its LP relaxation. If there is a one-to-one correspondence between the set of all possible elementary moves between feasible solutions of the MOCO problem and the edge-structure of $P$, i.e., solution $x_{1}$ can be obtained from $x_{2}$ by an elementary move if and only if the $x_{1}, x_{2}$-corresponding extreme points of $P$ are connected by an edge in $P$, then the resulting adjacency graphs for the combinatorial definition and the MILP-based definition of adjacency coincide.

Proof: Immediate consequence of Proposition 2.1. 


\section{Connectedness results for specific multiple objective com- binatorial optimization problems}

In this section, adjacency of efficient solutions is comprehensively investigated for various combinatorial optimization problems. Due to intended clarity and legibility, each of these fundamental problems is treated in a separate paragraph. On the one hand this list contains - to the best of our knowledge - all results available in the literature. The more significant part of this section yet contains two major components.

First, we investigate the question of adjacency of the graph of efficient solutions for problems which have not been treated in the literature so far. Second, the concept of adjacency of the graph of efficient solutions is extended and structural properties of this graph and its extensions are investigated. The latter is done exemplarily for MOSP problems. Treating all of the combinatorial optimization problems in this section likewise certainly goes beyond the scope of this work. Nevertheless, it should be emphasized that analogous results can be achieved for other problems utilizing similar techniques.

\subsection{Shortest path problems}

Let $G=(V, A)$ be a directed graph with source node $s$ and sink node $t$. The multiple objective shortest path problem (MOSP) can be formulated as

$$
\begin{array}{ll}
\min & \left(c^{1} x, \ldots, c^{p} x\right)^{T} \\
\text { s.t. } & \sum_{j=1}^{n} x_{i j}-\sum_{j=1}^{n} x_{j i}=\left\{\begin{aligned}
1, & \text { if } i=s \\
0, & \text { if } i \in\{1, \ldots, n\} \backslash\{s, t\} \\
-1, & \text { if } i=t .
\end{aligned}\right.
\end{array}
$$

Ehrgott and Klamroth (1997) called two efficient paths adjacent if they correspond to two adjacent basic feasible solutions of the linear program (5). Gorski (2004) showed that this LP formulation is appropriate in the sense of Definition 2.3. Furthermore, in Ehrgott and Klamroth (1997) it is shown that every given graph $G=(V, A)$ with cost vectors $c^{1}, \ldots, c^{p}: A \rightarrow \mathbb{R}_{+}$can be extended in such a way that the adjacency graph of the MOSP on the extended graph is not connected.

For this problem, a combinatorial definition of adjacency can be derived which is equivalent to the MILP-based definition. Paths are associated with flows and the residual flow of two paths is used to decide whether they are adjacent. A shortest path $P_{1}$ is adjacent to shortest path $P_{2}$ if the symmetric difference of their arc set in the residual graph corresponds to a single cycle. Note that these definitions are canonical extensions of the single objective case in the sense of Section 2.2. 


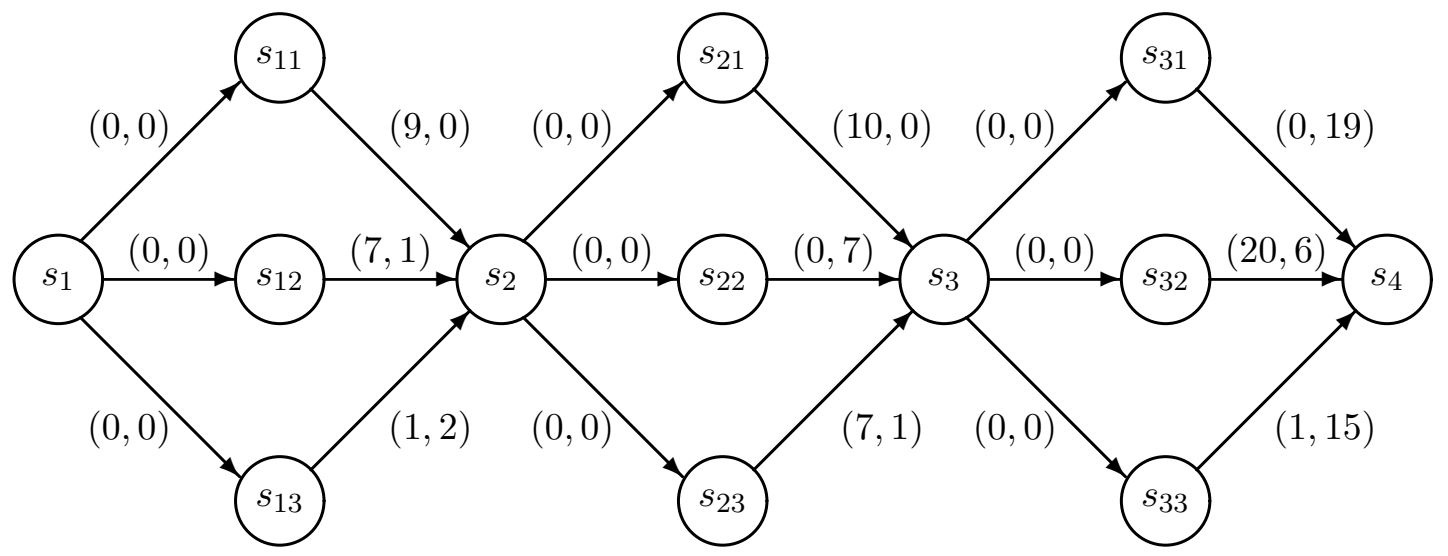

Figure 2: Digraph from Ehrgott and Klamroth (1997)

In Figure 2 the digraph used in Ehrgott and Klamroth (1997) is depicted. All efficient paths are indicated in Figure 3 together with their cost values. It is easy to verify that $P_{8}$ is not connected to any other efficient path. The adjacency graph has two connected components, $\left\{P_{8}\right\}$ being a singleton and $\left\{P_{i}: 1 \leq i \leq 12, i \neq 8\right\}$. This implies the following result.

Proposition 3.1 (Ehrgott and Klamroth (1997)) The adjacency graphs of efficient shortest paths are non-connected in general.

In the example of Ehrgott and Klamroth (1997) the set of weakly efficient solutions is connected. Consequently, investigating the adjacency graph of weakly efficient solutions suggests itself. A slight modification of the previous example, depicted in Figure 4, proves that this definition does also not result in a connected adjacency graph in general.

Proposition 3.2 The adjacency graphs of weakly efficient shortest paths are nonconnected in general.

In all examples so far, only two connected components of the adjacency graphs exist. One of them consists of a single element, while the second comprises all other (weakly) efficient solutions. Yet in general, we can derive the following structural property.

Proposition 3.3 In general, the number of connected components and the cardinality of the components are exponentially large in the size of the input data.

Proof: Suppose we have $k$ copies of the graph shown in Figure 4 . The cost vectors of copy $k$ are multiplied by the factor $100^{k}$. These $k$ copies are connected sequentially by 


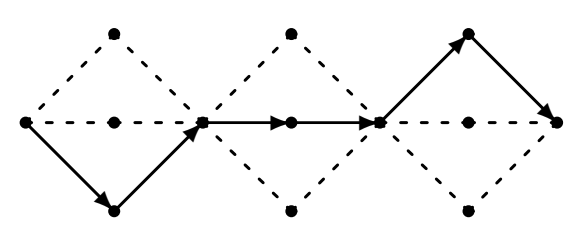

$P_{1}:(1,28)$

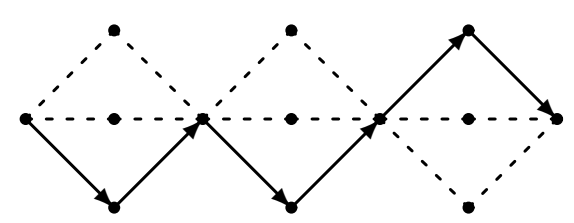

$P_{3}:(8,22)$

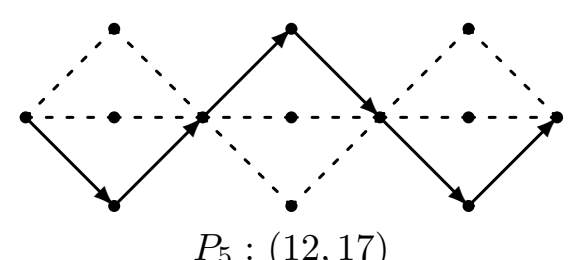

$P_{5}:(12,17)$

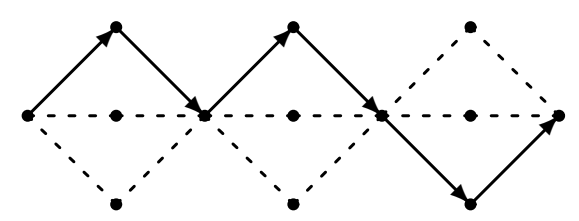

$P_{7}:(20,15)$

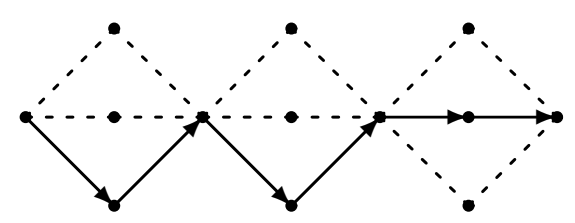

$P_{9}:(28,9)$

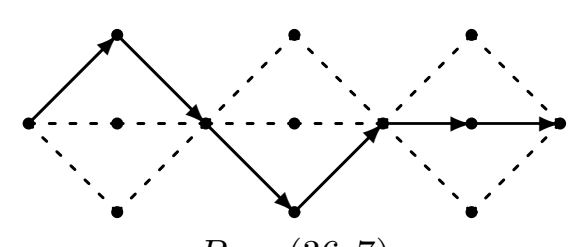

$P_{11}:(36,7)$

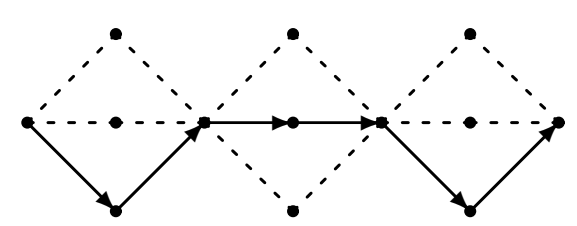

$P_{2}:(2,24)$

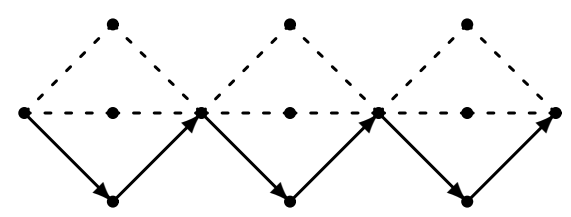

$P_{4}:(9,18)$

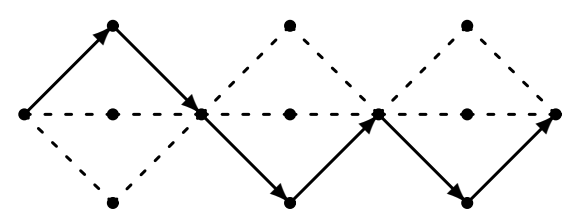

$P_{6}:(17,16)$

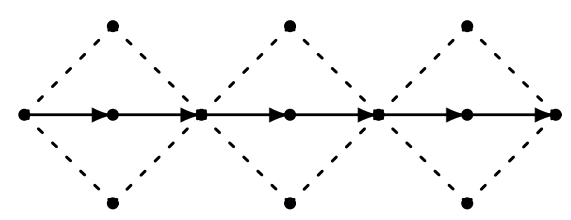

$P_{8}:(27,14)$

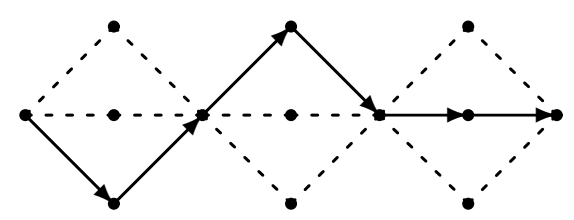

$P_{10}:(31,8)$

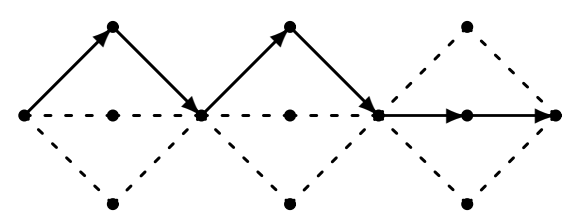

$P_{12}:(39,6)$

Figure 3: All efficient shortest paths for the example shown in Figure 2 and their objective vectors 


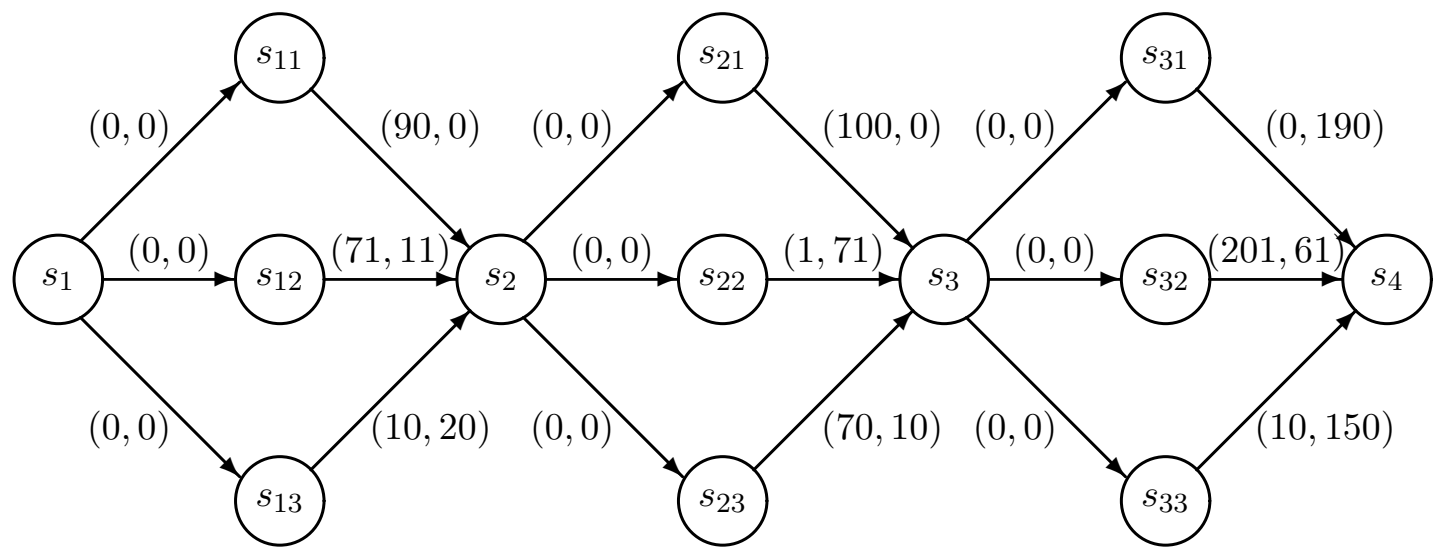

Figure 4: Modified digraph from Ehrgott and Klamroth (1997)

connecting node $s_{4}$ of copy $i, i=1, \ldots, k-1$, with node $s_{1}$ of copy $i+1$ using an arc with costs $(0,0)$. The resulting adjacency graph has $(19 \cdot k-1)$ arcs and $2^{k}$ different connected components. The largest component subsumes $11^{k}$ efficient solutions, the second largest $11^{k-1}$ efficient solutions, and so on.

\subsection{Minimum spanning tree problems}

Let $G=(V, A)$ be an undirected graph with $|V|=n$ nodes, and denote by $A(S):=\{a=$ $[i, j] \in A: i, j \in S\}$ the subset of edges in the subgraph of $G$ induced by $S \subseteq V$. The multiple objective spanning tree (MOST) problem can be formulated as

$$
\begin{array}{ll}
\min & \left(c^{1} x, \ldots, c^{p} x\right)^{T} \\
\text { s.t. } & \sum_{a \in A} x_{a}=n-1 \\
\sum_{a \in A(S)} x_{a} & \leq|S|-1 \quad \forall S \subseteq V \\
x_{a} & \in\{0,1\} .
\end{array}
$$

In Ehrgott and Klamroth (1997) a combinatorial definition of adjacency for efficient spanning trees was considered. Two spanning trees are adjacent if they have $n-2 \operatorname{arcs}$ in common. Non-connectivity of the adjacency graph was proven in this case also with the help of the graph of Figure 4, as there exists a one-to-one correspondence between efficient shortest paths and efficient spanning trees. It was also shown that every given graph can be extended in such a way that the adjacency graph for the multiple objective spanning tree problem in the new graph is non-connected. Gorski (2004) showed that the 
MILP formulation (6) is appropriate in the sense of Definition 2.3. The counter-example of Ehrgott and Klamroth (1997) was used to prove that the adjacency graph of MOST is also non-connected in this case.

Proposition 3.4 (Ehrgott and Klamroth (1997)) The adjacency graphs considered above for the multiple objective spanning tree problem are non-connected in general.

Note that for the spanning tree problem there exists a subclass of problems where the adjacency graph for both the combinatorial and the MILP-based definition of adjacency is always connected. This subclass is the set of all graphs which contain exactly one cycle.

\subsection{Minimum cost flow problems}

Let $G=(V, A)$ be a directed graph with capacities $u_{i j} \geq 0$ for every edge $(i, j) \in A$ and supply / demand values $b_{i}$ for every node $i \in V$. The multiple objective minimum cost flow problem (MOMC) can be formulated as

$$
\begin{array}{ll}
\min & \left(c^{1} x, \ldots, c^{p} x\right)^{T} \\
\text { s.t. } & \sum_{\{j:(i, j) \in A\}} x_{i j}-\sum_{\{j:(j, i) \in A\}} x_{j i}=b_{i} \quad \forall i \in N \\
0 \leq x_{i j} \leq u_{i j} & \forall(i, j) \in A .
\end{array}
$$

For the MOMC two efficient solutions are said to be adjacent if there exists a pivot operation between two bases corresponding to these solutions or, equivalently, if two spanning trees representing the solutions exist which differ by one edge only. This definition of adjacency is an extension of the definition for the shortest path problem and the spanning tree problem. Using the counter-example of Ehrgott and Klamroth (1997) and arguing that the shortest path problem is a particular case of the minimum cost flow problem, Przybylski et al. (2006) conclude that the adjacency graph of the minimum cost flow problem is not connected in general.

\subsection{Optimization problems on matroids}

A natural, combinatorial definition of adjacency for matroids is to call two solutions (consisting of $n$ elements each) adjacent if they have $n-1$ elements in common. Since the MOST is an example for a multiple objective minimization problem on a matroid for which we have shown non-connectedness with respect to this definition of adjacency in Section 3.1, we can conclude that the adjacency graph of such problems is in general non-connected. 


\subsection{Binary knapsack problems}

For the $\{0,1\}$-knapsack problem some results concerning the connectedness of the set of efficient solutions can be found in the recent literature. In da Silva et al. (2004), three different models of $\{0,1\}$-knapsack problems were studied and some connectedness results using an MILP-based definition of adjacency were presented for very specific problem classes. O'Sullivan and Walker (2004) proposed two algorithms for the equally-weighted bicriteria knapsack problem using a combinatorial definition of adjacency. These algorithms are only guaranteed to find the set of all efficient solutions under the assumption that this set is connected. We review the ideas of these two papers and show that the set of efficient solutions is in general non-connected neither in the sense of adjacency in da Silva et al. (2004) nor in the sense of adjacency in O'Sullivan and Walker (2004).

We consider a special class of $\{0,1\}$-knapsack problems with equal weights and bounded cardinality, i.e.,

$$
\begin{array}{ll}
\max & \left(c^{1} x, c^{2} x\right)^{T} \\
\text { s.t. } & \sum_{i=1}^{n} x_{i}=k \\
& x_{i} \in\{0,1\}, i=1 \ldots, n,
\end{array}
$$

where $c_{i}^{j} \geq 0$ represents the value of item $i$ on criterion $j, k \in \mathbb{N}$ with $k \leq n$ denotes the number of items that can be selected, and variables $x_{i}=1$ if and only if item $i$ is included in the knapsack. Let $K P(n, k)$ denote an instance of (8). Obviously, this problem has $\left(\begin{array}{l}n \\ k\end{array}\right)$ feasible solutions. As mentioned in da Silva et al. (2004), (8) can be relaxed to the case that at most $k$ items have to be chosen. Since all item values are non-negative, every efficient solution will have maximum cardinality.

We start our analysis with a combinatorial definition of adjacency which is also used in O'Sullivan and Walker (2004).

Definition 3.1 Two efficient knapsacks $x=\left(x_{1}, \ldots, x_{n}\right)^{T}$ and $x^{\prime}=\left(x_{1}^{\prime}, \ldots, x_{n}^{\prime}\right)^{T}$ of $K P(n, k)$ are called adjacent if $x^{\prime}$ can be obtained from $x$ by replacing one item in $x$ with one item of $x^{\prime}$ which is not contained in $x$.

Note that this elementary move is canonical. Two efficient knapsacks $x$ and $x^{\prime}$ are adjacent if and only if $\sum_{i=1}^{n}\left|x_{i}-x_{i}^{\prime}\right|=2$, i.e., if their Hamming distance is 2 . For $n \in\{1,2,3,4\}$ or $k \in\{0,1, n-1, n\}$ it is easy to see that $K P(n, k)$ has a connected adjacency graph.

Proposition 3.5 The adjacency graph of $K P(n, k)$ is connected for $n \in\{1,2,3,4\}$ or $k \in\{0,1, n-1, n\}$. 
In da Silva et al. (2004) another sufficient condition yielding a connected adjacency graph is specified.

Proposition 3.6 (da Silva et al. (2004)) Let an instance $K P(n, k)$ be given such that $c_{i}^{1}+c_{i}^{2}=\alpha$ for all $i=1, \ldots, n$ and for some $\alpha \in \mathbb{N}$. Then all $\left(\begin{array}{l}n \\ k\end{array}\right)$ feasible solutions are efficient solutions of (8) and hence, the adjacency graph of the problem is connected.

Unfortunately, this connectedness result is no longer valid for the general case.

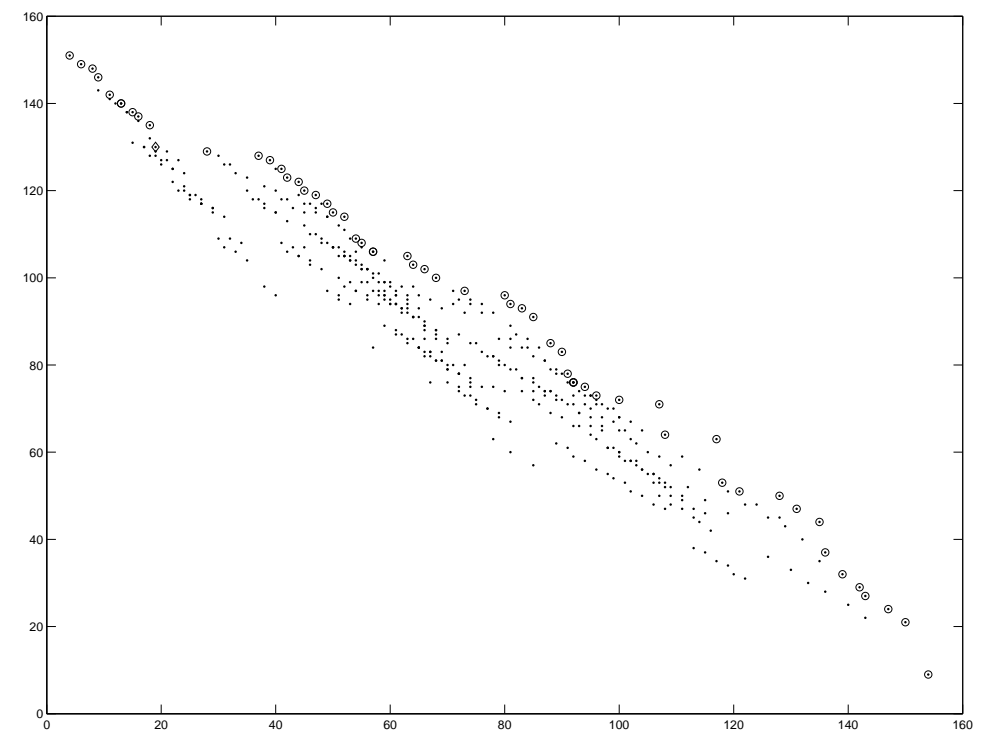

Figure 5: Nondominated set of the non-connected example problem used in the proof of Proposition 3.7. The nondominated set consists of two connected components, one indicated by circles, the other - a singleton - indicated by a diamond.

Proposition 3.7 The adjacency graph of a $\{0,1\}$-knapsack problem of the form (8) with adjacency defined as in Definition 3.1 is non-connected in general.

Proof: Consider $K P(15,3)$ with the objective function vectors

$$
\left(\begin{array}{l}
c^{1} x \\
c^{2} x
\end{array}\right)=\left(\begin{array}{rrrrrrrrrrrrrrr}
55 & 51 & 48 & 44 & 37 & 36 & 27 & 16 & 14 & 10 & 8 & 5 & 3 & 1 & 0 \\
0 & 3 & 6 & 18 & 19 & 26 & 27 & 28 & 29 & 39 & 41 & 47 & 49 & 50 & 52
\end{array}\right) .
$$

The problem has 455 feasible and 59 efficient solutions (cf. Figure 5). All efficient solutions $S_{1}, \ldots, S_{59}$ and their corresponding objective function vectors are listed in Table 1. Using the plotted boxes it is easy to verify that the efficient solution $S_{11}$ is not adjacent to any 


\begin{tabular}{|c|c|c|c|c|c|c|c|c|c|c|c|c|c|c|c|c|c|}
\hline \multicolumn{2}{|c|}{ Cx $x$} & \multicolumn{15}{|c|}{$x$} & $S$ \\
\hline 4 & 151 & 0 & 0 & 0 & 0 & 0 & 0 & 0 & 0 & 0 & 0 & 0 & 0 & 1 & 1 & 1 & $S_{1}$ \\
\hline 6 & 149 & 0 & 0 & 0 & 0 & 0 & 0 & 0 & 0 & 0 & 0 & 0 & 1 & 0 & 1 & 1 & $S_{2}$ \\
\hline 8 & 148 & 0 & 0 & 0 & 0 & 0 & 0 & 0 & 0 & 0 & 0 & 0 & 1 & 1 & 0 & 1 & $S_{3}$ \\
\hline 9 & 146 & 0 & 0 & 0 & 0 & 0 & 0 & 0 & 0 & 0 & 0 & 0 & 1 & 1 & 1 & 0 & $S_{4}$ \\
\hline 11 & 142 & 0 & 0 & 0 & 0 & 0 & 0 & 0 & 0 & 0 & 0 & 1 & 0 & 1 & 0 & 1 & $S_{5}$ \\
\hline 13 & 140 & 0 & 0 & 0 & 0 & 0 & 0 & 0 & 0 & 0 & 1 & 0 & 0 & 1 & 0 & 1 & $S_{6}$ \\
\hline 13 & 140 & 0 & 0 & 0 & 0 & 0 & 0 & 0 & 0 & 0 & 0 & 1 & 1 & 0 & 0 & 1 & $S_{7}$ \\
\hline 15 & 138 & 0 & 0 & 0 & 0 & 0 & 0 & 0 & 0 & 0 & 1 & 0 & 1 & 0 & 0 & 1 & $S_{8}$ \\
\hline 16 & 137 & 0 & 0 & 0 & 0 & 0 & 0 & 0 & 0 & 0 & 0 & 1 & 1 & 1 & 0 & 0 & $S_{9}$ \\
\hline 18 & 135 & 0 & 0 & 0 & 0 & 0 & 0 & 0 & 0 & 0 & 1 & 0 & 1 & 1 & 0 & 0 & $S_{10}$ \\
\hline 19 & 130 & 0 & 0 & 0 & 0 & 0 & 0 & 0 & 0 & 0 & 1 & 1 & 0 & 0 & 1 & 0 & $S_{11}$ \\
\hline 28 & 129 & 0 & 0 & 0 & 0 & 0 & 0 & 1 & 0 & 0 & 0 & 0 & 0 & 0 & 1 & 1 & $S_{12}$ \\
\hline 37 & 128 & 0 & 0 & 0 & 0 & 0 & 1 & 0 & 0 & 0 & 0 & 0 & 0 & 0 & 1 & 1 & $S_{13}$ \\
\hline 39 & 127 & 0 & 0 & 0 & 0 & 0 & 1 & 0 & 0 & 0 & 0 & 0 & 0 & 1 & 0 & 1 & $S_{14}$ \\
\hline 41 & 125 & 0 & 0 & 0 & 0 & 0 & 1 & 0 & 0 & 0 & 0 & 0 & 1 & 0 & 0 & 1 & $S_{15}$ \\
\hline 42 & 123 & 0 & 0 & 0 & 0 & 0 & 1 & 0 & 0 & 0 & 0 & 0 & 1 & 0 & 1 & 0 & $S_{16}$ \\
\hline 44 & 122 & 0 & 0 & 0 & 0 & 0 & 1 & 0 & 0 & 0 & 0 & 0 & 1 & 1 & 0 & 0 & $S_{17}$ \\
\hline 45 & 120 & 0 & 0 & 0 & 1 & 0 & 0 & 0 & 0 & 0 & 0 & 0 & 0 & 0 & 1 & 1 & $S_{18}$ \\
\hline 47 & 119 & 0 & 0 & 0 & 1 & 0 & 0 & 0 & 0 & 0 & 0 & 0 & 0 & 1 & 0 & 1 & $S_{19}$ \\
\hline 49 & 117 & 0 & 0 & 0 & 1 & 0 & 0 & 0 & 0 & 0 & 0 & 0 & 1 & 0 & 0 & 1 & $S_{20}$ \\
\hline 50 & 115 & 0 & 0 & 0 & 1 & 0 & 0 & 0 & 0 & 0 & 0 & 0 & 1 & 0 & 1 & 0 & $S_{21}$ \\
\hline 52 & 114 & 0 & 0 & 0 & 1 & 0 & 0 & 0 & 0 & 0 & 0 & 0 & 1 & 1 & 0 & 0 & $S_{22}$ \\
\hline 54 & 109 & 0 & 0 & 0 & 1 & 0 & 0 & 0 & 0 & 0 & 1 & 0 & 0 & 0 & 0 & 1 & $S_{23}$ \\
\hline 55 & 108 & 0 & 0 & 0 & 1 & 0 & 0 & 0 & 0 & 0 & 0 & 1 & 0 & 1 & 0 & 0 & $S_{24}$ \\
\hline 57 & 106 & 0 & 0 & 0 & 1 & 0 & 0 & 0 & 0 & 0 & 1 & 0 & 0 & 1 & 0 & 0 & $S_{25}$ \\
\hline 57 & 106 & 0 & 0 & 0 & 1 & 0 & 0 & 0 & 0 & 0 & 0 & 1 & 1 & 0 & 0 & 0 & $S_{26}$ \\
\hline 63 & 105 & 0 & 0 & 0 & 0 & 0 & 1 & 1 & 0 & 0 & 0 & 0 & 0 & 0 & 0 & 1 & $S_{27}$ \\
\hline 64 & 103 & 0 & 0 & 0 & 0 & 0 & 1 & 1 & 0 & 0 & 0 & 0 & 0 & 0 & 1 & 0 & $S_{28}$ \\
\hline 66 & 102 & 0 & 0 & 0 & 0 & 0 & 1 & 1 & 0 & 0 & 0 & 0 & 0 & 1 & 0 & 0 & $S_{29}$ \\
\hline 68 & 100 & 0 & 0 & 0 & 0 & 0 & 1 & 1 & 0 & 0 & 0 & 0 & 1 & 0 & 0 & 0 & $S_{30}$ \\
\hline 73 & 97 & 0 & 0 & 0 & 0 & 1 & 1 & 0 & 0 & 0 & 0 & 0 & 0 & 0 & 0 & 1 & $S_{31}$ \\
\hline 80 & 96 & 0 & 0 & 0 & 1 & 0 & 1 & 0 & 0 & 0 & 0 & 0 & 0 & 0 & 0 & 1 & $S_{32}$ \\
\hline 81 & 94 & 0 & 0 & 0 & 1 & 0 & 1 & 0 & 0 & 0 & 0 & 0 & 0 & 0 & 1 & 0 & $S_{33}$ \\
\hline 83 & 93 & 0 & 0 & 0 & 1 & 0 & 1 & 0 & 0 & 0 & 0 & 0 & 0 & 1 & 0 & 0 & $S_{34}$ \\
\hline 85 & 91 & 0 & 0 & 0 & 1 & 0 & 1 & 0 & 0 & 0 & 0 & 0 & 1 & 0 & 0 & 0 & $S_{35}$ \\
\hline 88 & 85 & 0 & 0 & 0 & 1 & 0 & 1 & 0 & 0 & 0 & 0 & 1 & 0 & 0 & 0 & 0 & $S_{36}$ \\
\hline 90 & 83 & 0 & 0 & 0 & 1 & 0 & 1 & 0 & 0 & 0 & 1 & 0 & 0 & 0 & 0 & 0 & $S_{37}$ \\
\hline 91 & 78 & 1 & 0 & 0 & 0 & 0 & 1 & 0 & 0 & 0 & 0 & 0 & 0 & 0 & 0 & 1 & $S_{38}$ \\
\hline 92 & 76 & 1 & 0 & 0 & 0 & 0 & 1 & 0 & 0 & 0 & 0 & 0 & 0 & 0 & 1 & 0 & $S_{39}$ \\
\hline 92 & 76 & 0 & 0 & 1 & 1 & 0 & 0 & 0 & 0 & 0 & 0 & 0 & 0 & 0 & 0 & 1 & $S_{40}$ \\
\hline 92 & 76 & 0 & 1 & 0 & 0 & 0 & 1 & 0 & 0 & 0 & 0 & 0 & 1 & 0 & 0 & 0 & $S_{41}$ \\
\hline 94 & 75 & 1 & 0 & 0 & 0 & 0 & 1 & 0 & 0 & 0 & 0 & 0 & 0 & 1 & 0 & 0 & $S_{42}$ \\
\hline 96 & 73 & 1 & 0 & 0 & 0 & 0 & 1 & 0 & 0 & 0 & 0 & 0 & 1 & 0 & 0 & 0 & $S_{43}$ \\
\hline 100 & 72 & 0 & 0 & 0 & 0 & 1 & 1 & 1 & 0 & 0 & 0 & 0 & 0 & 0 & 0 & 0 & $S_{44}$ \\
\hline 107 & 71 & 0 & 0 & 0 & 1 & 0 & 1 & 1 & 0 & 0 & 0 & 0 & 0 & 0 & 0 & 0 & $S_{45}$ \\
\hline 108 & 64 & 0 & 0 & 0 & 1 & 1 & 0 & 1 & 0 & 0 & 0 & 0 & 0 & 0 & 0 & 0 & $S_{46}$ \\
\hline 117 & 63 & 0 & 0 & 0 & 1 & 1 & 1 & 0 & 0 & 0 & 0 & 0 & 0 & 0 & 0 & 0 & $S_{47}$ \\
\hline 118 & 53 & 1 & 0 & 0 & 0 & 0 & 1 & 1 & 0 & 0 & 0 & 0 & 0 & 0 & 0 & 0 & $S_{48}$ \\
\hline 121 & 51 & 0 & 0 & 1 & 0 & 1 & 1 & 0 & 0 & 0 & 0 & 0 & 0 & 0 & 0 & 0 & $S_{49}$ \\
\hline 128 & 50 & 0 & 0 & 1 & 1 & 0 & 1 & 0 & 0 & 0 & 0 & 0 & 0 & 0 & 0 & 0 & $S_{50}$ \\
\hline 131 & 47 & 0 & 1 & 0 & 1 & 0 & 1 & 0 & 0 & 0 & 0 & 0 & 0 & 0 & 0 & 0 & $S_{51}$ \\
\hline 135 & 44 & 1 & 0 & 0 & 1 & 0 & 1 & 0 & 0 & 0 & 0 & 0 & 0 & 0 & 0 & 0 & $S_{52}$ \\
\hline 136 & 37 & 1 & 0 & 0 & 1 & 1 & 0 & 0 & 0 & 0 & 0 & 0 & 0 & 0 & 0 & 0 & $S_{53}$ \\
\hline 139 & 32 & 1 & 0 & 1 & 0 & 0 & 1 & 0 & 0 & 0 & 0 & 0 & 0 & 0 & 0 & 0 & $S_{54}$ \\
\hline 142 & 29 & 1 & 1 & 0 & 0 & 0 & 1 & 0 & 0 & 0 & 0 & 0 & 0 & 0 & 0 & 0 & $S_{55}$ \\
\hline 143 & 27 & 0 & 1 & 1 & 1 & 0 & 0 & 0 & 0 & 0 & 0 & 0 & 0 & 0 & 0 & 0 & $S_{56}$ \\
\hline 147 & 24 & 1 & 0 & 1 & 1 & 0 & 0 & 0 & 0 & 0 & 0 & 0 & 0 & 0 & 0 & 0 & $S_{57}$ \\
\hline 150 & 21 & 1 & 1 & 0 & 1 & 0 & 0 & 0 & 0 & 0 & 0 & 0 & 0 & 0 & 0 & 0 & $S_{58}$ \\
\hline 154 & 9 & 1 & 1 & 1 & 0 & 0 & 0 & 0 & 0 & 0 & 0 & 0 & 0 & 0 & 0 & 0 & $S_{59}$ \\
\hline
\end{tabular}

Table 1: All efficient solutions of the example used in the proof of Proposition 3.7. 
other solution in the sense of Definition 3.1. Consequently, the adjacency graph of the given problem which can be seen in Figure 6 is non-connected.

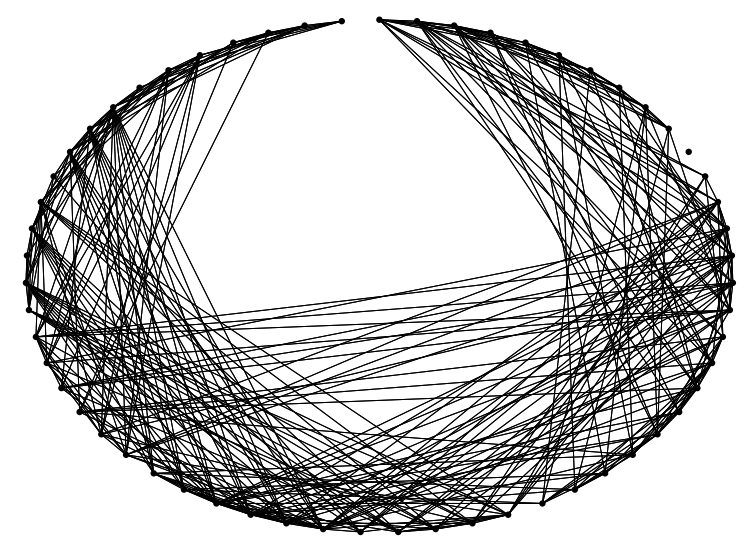

Figure 6: Adjacency graph of the non-connected example problem used in the proof of Proposition 3.7.

Corollary 3.1 The algorithms proposed by O'Sullivan and Walker (2004) for solving the $\{0,1\}$-knapsack problem with equal weights and bounded cardinality fail to compute the set of efficient solutions in general.

In Section 4, we report about numerical results indicating the likelihood that a nonconnected adjacency graph of problem (8) appears in randomly generated instances. Note that for these investigations problems $K P(n, k)$ with $k>\frac{n}{2}$ are not of interest as they can be transformed into an equivalent knapsack problem where the decision is which objects to leave out of a knapsack. The resulting problem can be interpreted as $K P(n, \tilde{k})$ for $\tilde{k} \leq \frac{n}{2}$.

Next, we concentrate on the MILP-based definition of adjacency which is considered in da Silva et al. (2004). Since the MILP formulation (8) is canonical it can be extended to an appropriate MILP formulation using the proof of Lemma 2.1. Let $P:=\left\{x \in[0,1]^{n}\right.$ : $\left.\sum_{i=1}^{n} x_{i}=k\right\}$ denote the feasible set of the LP relaxation of (8).

Lemma 3.1 Let $n \geq 5$. Two extreme points $u$ and $v$ of the $\{0,1\}$-knapsack polytope $P=\left\{x \in[0,1]^{n}: \sum_{i=1}^{n} x_{i}=k\right\}$ are connected by an edge if and only if $u$ and $v$ are adjacent in the sense of Definition 3.1 .

Proof: According to Geist and Rodin (1992) it suffices to show that two extreme points $u$ and $v$ of $P$ are connected by an edge if and only if there does not exist two other extreme 
points $w^{1}$ and $w^{2}$ of $P$, i.e., other feasible solutions of $K P(n, k)$, such that

$$
\frac{1}{2}\left(w^{1}+w^{2}\right)=\frac{1}{2}(u+v) .
$$

First, let two feasible solutions $u$ and $v$ of $K P(n, k)$ be given that are adjacent according to Definition 3.1. By definition they differ in exactly one item in the knapsack. Without loss of generality we assume that $u_{1}=v_{2}=1, u_{2}=v_{1}=0$ and $u_{i}=v_{i}$ for all $i=3, \ldots, n$. Suppose $u$ and $v$ are not connected by an edge in $P$, i.e., there exist two other feasible solutions $w^{1}$ and $w^{2}$ satisfying equation (9). Since $u$ and $v$ are equal starting from the third component and thus $u_{i}=v_{i}=0$ or $u_{i}=v_{i}=1$ for $i=3, \ldots, n, \frac{1}{2}\left(u_{i}+v_{i}\right)$ equals either 0 or 1 and hence $w_{i}^{1}=w_{i}^{2}=u_{i}=v_{i}$ for all $i=3, \ldots, n$ must hold to satisfy (9). So, $w^{1}$ and $w^{2}$ can differ from $u$ and $v$ only in the first two components which means that either $w_{1}^{j}=w_{2}^{j}=0$ or $w_{1}^{j}=w_{2}^{j}=1(j \in\{1,2\})$, which is impossible due to the constraint $\sum_{i=1}^{n} w_{i}^{j}=k$. Hence, $u$ and $v$ are connected by an edge in $P$.

Now let $u$ and $v$ be not adjacent solutions in the sense of Definition 3.1. Then $u$ and $v$ differ in at least two different items in each knapsack. Without loss of generality we assume that the first and the second item is contained in $u$ but not in $v$ and the third and the fourth item is contained $v$ but not in $u$. Now define

$$
w_{i}^{1}=\left\{\begin{array}{l}
1, \text { if } i \in\{1,3\} \\
0, \text { if } i \in\{2,4\} \\
u_{i}, \text { if } i \geq 5
\end{array} \quad \text { and } w_{i}^{2}=\left\{\begin{array}{l}
1, \text { if } i \in\{2,4\} \\
0, \text { if } i \in\{1,3\} \\
v_{i}, \text { if } i \geq 5
\end{array}\right.\right.
$$

Then, $w^{1}$ and $w^{2}$ are feasible and both different from $u$ and $v$. Equation (9) is satisfied and hence $u$ and $v$ are not connected by an edge in $P$.

According to Lemma 3.1, the adjacency structure of the efficient extreme points of $P$ coincides with the adjacency structure induced by Definition 3.1. Hence, the adjacency graph with respect to the appropriate MILP formulation based on (8) and the adjacency graph resulting from Definition 3.1 are the same (cf. Proposition 2.2). Thus, Proposition 3.7 immediately implies the following result.

Corollary 3.2 In general, the set of efficient solutions of $K P(n, k)$ is non-connected with respect to the appropriate MILP formulation based on (8).

Finally we investigate a combinatorial definition of adjacency for another variant of the knapsack problem, namely the $\{0,1\}$-multiple choice knapsack problem with equal 
weights:

$$
\begin{array}{ll}
\max & \left(\sum_{i=1}^{n} \sum_{j=1}^{k_{i}} c_{i j}^{1} x_{i j}, \sum_{i=1}^{n} \sum_{j=1}^{k_{i}} c_{i j}^{2} x_{i j}\right)^{T} \\
\text { s.t. } & \sum_{j=1}^{k_{i}} x_{i j}=1, i=1, \ldots, n, \\
& x_{i j} \in\{0,1\}, i=1, \ldots, n, j=1, \ldots, k_{i} .
\end{array}
$$

The given problem can be interpreted as follows: Given $n$ disjoint baskets $B_{1}, \ldots, B_{n}$ each having exactly $k_{i}$ items, the objective is to maximize the overall profit with the restriction that exactly one item is chosen from each basket. Problem (10) is a more structured knapsack problem compared to Problem (8) since items cannot be combined arbitrarily. We consider the following combinatorial definition of adjacency.

Definition 3.2 Two efficient knapsacks $x$ and $x^{\prime}$ of the $\{0,1\}$-multiple choice knapsack problem with equal weights are called adjacent if $x^{\prime}$ and $x$ differ in one item in exactly one basket $B_{i}$ for an $i \in\{1, \ldots, n\}$.

This definition of adjacency is again canonical since for single objective problems, any maximal knapsack must contain an item with maximal profit from each basket. Alternative optimal solutions may exist if at least one basket contains more than one item with maximal profit. All these optimal solutions are adjacent in the sense of Definition 3.2.

In the multiple objective case the situation is, however, different. The counter-example of Ehrgott and Klamroth (1997) can be reformulated as a $\{0,1\}$-multiple choice knapsack problem with equal weights which implies the following non-connectedness result.

Proposition 3.8 The adjacency graph of a $\{0,1\}$-multiple choice knapsack problem with equal weights, where adjacency of two efficient solutions is defined according to Definition 3.2, is non-connected in general.

Proof: Consider the following modification of the non-connected example problem for the MOSP given in Ehrgott and Klamroth (1997) (see Figure 2). We redefine the resulting cost vectors $\left(c_{i j}^{1}, c_{i j}^{2}\right)^{T}$ for the three paths from the node $s_{i}$ to node $s_{i+1}$ via $s_{i j}$ by setting

$$
\tilde{c}_{i j}^{q}=\max \left\{c_{i j}^{q}: i, j=1,2,3 ; q=1,2\right\}-c_{i j}^{q}
$$

for $i, j=1,2,3$ and $q=1,2$ and interpret the new cost vectors of the three paths from the node $s_{i}$ to node $s_{i+1}$ as profit vectors for basket $B_{i}, i=1,2,3$. This results in the three baskets

$B_{1}=\left\{\left(\begin{array}{l}11 \\ 20\end{array}\right),\left(\begin{array}{l}13 \\ 19\end{array}\right),\left(\begin{array}{l}19 \\ 18\end{array}\right)\right\}, B_{2}=\left\{\left(\begin{array}{l}10 \\ 20\end{array}\right),\left(\begin{array}{l}20 \\ 13\end{array}\right),\left(\begin{array}{c}13 \\ 19\end{array}\right)\right\}, B_{3}=\left\{\left(\begin{array}{c}20 \\ 1\end{array}\right),\left(\begin{array}{c}0 \\ 14\end{array}\right),\left(\begin{array}{c}19 \\ 5\end{array}\right)\right\}$. 
Since we have transformed the minimization problem into a maximization problem by taking the negative value of each cost vector followed by a shift of these vectors by an amount of $\max \left\{c_{i j}^{q}\right\}=20$, Figure 3 still represents all efficient solutions of the modified problem. The profit vectors of the resulting solutions $K_{1}, \ldots, K_{12}$ are given by $(60,60)^{T}-$ $c\left(P_{i}\right)$ where $c\left(P_{i}\right)$ corresponds to the cost vector of $P_{i}$ in Figure 3 for $i=1, \ldots, 12$.

Items in at least two baskets have to be exchanged when transforming $K_{8}$ into $K_{j}$, $j \neq 8$ by elementary moves. Hence, $K_{8}$ is not adjacent to any other efficient solution in the sense of Definition 3.2.

In Section 4.2 we investigate the frequency with which a non-connected adjacency graph for problem (10) occurs empirically in randomly generated instances.

\subsection{General knapsack problems}

Since the general knapsack problem subsumes the $\{0,1\}$-knapsack problem with bounded cardinality discussed above as a special case and since we have shown the nonconnectedness of this problem, the general knapsack problem is in general non-connected as well if connectedness is defined, for example, based on elementary moves similar to Section 3.5 above.

\subsection{Integer programming problems with fixed (or bounded) cardinalities}

The same reasoning as above (Section 3.6) applies.

\subsection{Unconstrained binary optimization problems}

Since, in general, the adjacency graph for the $\{0,1\}$-knapsack problem with equal weights and bounded cardinality is non-connected for well-established definitions of adjacency of efficient solutions (see Section 3.5), we will focus on unconstrained $\{0,1\}$-problems in this subsection since these problems possess even less structure. Formally, an unconstrained $\{0,1\}$-problem is defined as follows:

$$
\begin{array}{ll}
\max & \left(c^{1} x, c^{2} x\right)^{T} \\
\text { s.t. } & x_{i} \in\{0,1\}, \quad i=1, \ldots, n .
\end{array}
$$

We assume without loss of generality that $c_{i}^{1} \cdot c_{i}^{2}<0$ (but not necessarily $c_{i}^{1}<0$ and $c_{i}^{2}>0$ ) for all $i=1, \ldots, n$. Otherwise either $x_{i}=0$ or $x_{i}=1$ in every efficient solution.

In problem (11) the number of variables set equal to one is not fixed. Consequently, an appropriate notion of adjacency is not evident. Nevertheless, Definition 3.2 can be 
transferred to this problem. Consider the following modified version of problem (11).

$$
\begin{array}{lll}
\max & \left(c^{1} x, c^{2} x\right)^{T} \\
\text { s.t. } & x_{i}+y_{i}=1, \quad i=1, \ldots, n \\
& x_{i}, y_{i} \in\{0,1\}, \quad i=1, \ldots, n .
\end{array}
$$

Clearly, either $x_{i}=1$ or $y_{i}=1$ and in each feasible solution vector $(x, y)^{T}$, the number of ones is exactly $n$, i.e., $\sum_{i=1}^{n}\left(x_{i}+y_{i}\right)=n$. Every solution of problem (12) has the same cardinality. However, the notion of adjacency for $\{0,1\}$-knapsack problems with fixed cardinality does not apply directly to problem (12) since the values of $x_{i}$ and $y_{i}, i=$ $1, \ldots, n$, cannot be chosen independently as they are coupled by a side constraint. By introducing additional zero cost vectors for each $y_{i}, i=1, \ldots, n,(12)$ can be interpreted as a $\{0,1\}$-multiple choice knapsack problem with equal weights where either $x_{i}$ or $y_{i}$ has to be included in the knapsack, $i=1, \ldots, n$. Hence, Definition 3.2 can be applied to (12). Since this definition of adjacency for the extended problem results in single ' 1 -to-0' or ' 0 -to- 1 ' swaps in exactly one $x_{i}$ in (11), we define:

Definition 3.3 Two efficient solutions $x$ and $x^{\prime}$ of the unconstrained $\{0,1\}$-problem are called adjacent if they differ in exactly one component, i.e., if $\sum_{i=1}^{n}\left|x_{i}-x_{i}^{\prime}\right|=1$.

If we extend the last definition to all $2^{n}$ feasible solutions of the problem which can be identified with the set of all extreme points of the $n$-dimensional unit cube $W:=[0,1]^{n}$, two feasible (efficient) solutions are adjacent if and only if they are connected by an edge in $W$. But since $W$ in combination with (11) can be easily modeled by an appropriate MILP formulation, the adjacency graph which results from Definition 3.3 coincides with the adjacency graph of this appropriate MILP formulation by Proposition 2.2.

Proposition 3.9 The adjacency graph of an unconstrained $\{0,1\}$-problem of the form (11), where adjacency of two efficient solutions is defined according to Definition 3.3, is non-connected in general.

Proof: Consider the following unconstrained $\{0,1\}$-problem with objective matrix

$$
C=\left(\begin{array}{rrrrrrrrrrrrr}
-126 & -121 & -120 & -103 & -100 & -97 & -51 & -29 & -19 & -18 & -17 & -13 & 124 \\
100 & 94 & 90 & 74 & 73 & 68 & 67 & 55 & 54 & 41 & 23 & 7 & -126
\end{array}\right) .
$$

The set of all efficient solutions of the problem consists of exactly 155 vectors. It can be shown that the efficient solution

$$
x=(0,1,0,1,1,1,1,1,1,1,0,1,0)^{T} \text { with objective value } C x=(-551,533)^{T}
$$


is not adjacent to any other efficient solution of the problem in the sense of Definition 3.3.

Corollary 3.3 Let an appropriate MILP formulation of problem (11) be given where the polytope of the LP relaxation describes the $n$-dimensional unit cube $[0,1]^{n}$. Then, the adjacency graph of the unconstrained $\{0,1\}$-problem with respect to the given MILP formulation is non-connected in general.

\subsection{Linear assignment problems}

We consider two definitions of adjacency for the linear assignment problem: A combinatorial definition based on swapping rows in the assignment matrix, and an MILP-based definition of adjacency. The bicriteria linear assignment problem can be formulated as

$$
\begin{array}{ll}
\min & \left(\sum_{i, j=1}^{n} c_{i j}^{1} x_{i j}, \sum_{i, j=1}^{n} c_{i j}^{2} x_{i j}\right)^{T} \\
\text { s.t. } & \sum_{i=1}^{n} x_{i j}=1, \quad j=1, \ldots, n \\
& \sum_{j=1}^{n} x_{i j}=1, \quad i=1, \ldots, n \\
& x_{i j} \in\{0,1\}, \quad i, j=1, \ldots, n
\end{array}
$$

with objective coefficients $c_{i j}^{1}, c_{i j}^{2} \geq 0$ for all $i, j=1, \ldots, n$.

First we consider an intuitive combinatorial definition of adjacency based on a simple swap of two rows of the assignment matrix. This definition is not canonical, i.e., it does not yield a connected graph of optimal solutions for the single objective version of the problem:

Proposition 3.10 Swapping two rows of the assignment matrix of a single objective linear assignment problem without changing the objective function value does in general not permit to construct the complete set of optimal solutions starting from an arbitrary optimal solution.

Proof: Consider a single objective linear assignment problem with $n=4$ and cost matrix

$$
C=\left(c_{i j}^{1}\right)_{i, j=1, \ldots, n}=\left(\begin{array}{cccc}
1 & \infty & 1 & \infty \\
1 & 1 & \infty & \infty \\
\infty & \infty & 1 & 1 \\
\infty & 1 & \infty & 1
\end{array}\right)
$$


This problem has two optimal assignments with value 4 :

Assignment 1: $x_{11}=x_{22}=x_{33}=x_{44}=1$ and $x_{i j}=0$ otherwise.

Assignment 2: $x_{13}=x_{21}=x_{34}=x_{42}=1$ and $x_{i j}=0$ otherwise.

Clearly, these assignments cannot be obtained from each other by one single row swap.

Turning our attention to an MILP-based definition of adjacency using formulation (13), we observe that the bicriteria linear assignment problem is a special case of the minimum cost flow problem (cf. Section 3.3). Thus, we can expect the existence of a canonical definition of adjacency in this case. The matrix describing the assignment polytope is totally unimodular (see, for example, Nemhauser and Wolsey, 1999) and hence formulation (13) is an appropriate MILP formulation. To simplify the discussion, we use the following combinatorial interpretation of the resulting concept of adjacency.

Definition 3.4 Let $G=\left(V_{1} \cup V_{2}, A\right)$ with $\left|V_{1}\right|=\left|V_{2}\right|=n$ be a bipartite graph with edge costs $c^{1}, c^{2}: A \rightarrow \mathbb{R}$ that models a given instance of the bicriteria linear assignment problem, and let $A_{1}$ and $A_{2}$ be the edges selected in two different assignments. We call the solutions corresponding to $A_{1}$ and $A_{2}$ adjacent if the graph induced by $A_{1} \cup A_{2}$ contains exactly one cycle.

According to Balinski and Russakoff (1974), this combinatorial definition of adjacency corresponds to the MILP-based definition of adjacency induced by the assignment polytope $P$. Equivalently, two assignments $A_{1}$ and $A_{2}$ are adjacent if and only if their symmetric difference $A_{1} \triangle A_{2}=\left(A_{1} \cup A_{2}\right) \backslash\left(A_{1} \cap A_{2}\right)$ consists of exactly one cycle the edges of which alternately belong to $A_{1}$ and $A_{2}$, respectively (Hausmann, 1980).

Since any pair of vertices of the assignment polytope is connected by a path of length less than or equal to 2 (see, for example, Balinski and Russakoff, 1974; Hausmann, 1980), the generalized adjacency graph $\mathcal{G}_{2}^{\prime}$ of problem (13) is always connected. However, if we restrict ourselves to direct adjacency according to Definition 3.4, the adjacency graph $\mathcal{G}=\mathcal{G}_{1}^{\prime}$ of the bicriteria linear assignment problem is non-connected in general.

Proposition 3.11 The adjacency graph $\mathcal{G}$ of the bicriteria linear assignment problem using Definition 3.4 for characterizing adjacent assignments is not connected in general.

Proof: We modify the counter-example of Ehrgott and Klamroth (1997). Let the following 


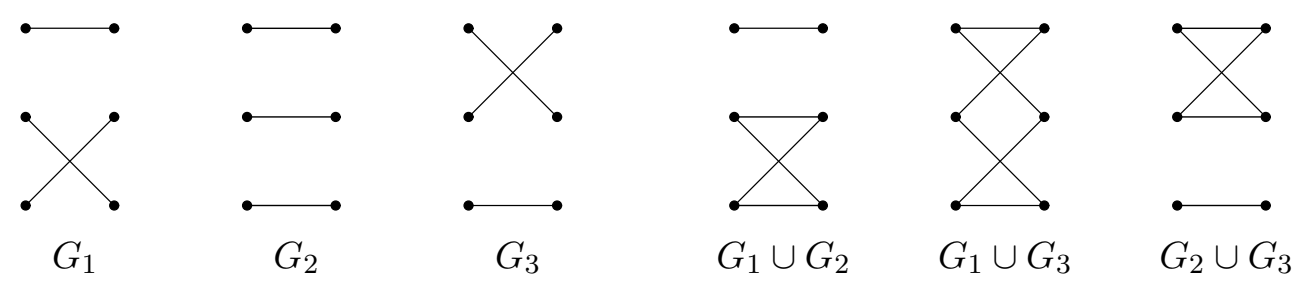

Figure 7: All feasible assignments with finite costs for the subproblems $S_{i}$ in the proof of Proposition 3.11 and their pairwise union.

six cost-submatrices of a $(9 \times 9)$ bicriteria linear assignment problem be given by $\begin{array}{ll}C_{(1: 3,1: 3)}^{1}=\left(\begin{array}{ccc}0 & 0 & \infty \\ 1 & 7 & 0 \\ \infty & 9 & 0\end{array}\right), C_{(4: 6,4: 6)}^{1}=\left(\begin{array}{ccc}0 & 0 & \infty \\ 7 & 0 & 0 \\ \infty & 10 & 0\end{array}\right), C_{(7: 9,7: 9)}^{1}=\left(\begin{array}{ccc}0 & 0 & \infty \\ 1 & 20 & 0 \\ \infty & 0 & 0\end{array}\right), \\ C_{(1: 3,1: 3)}^{2}=\left(\begin{array}{ccc}0 & 0 & \infty \\ 2 & 1 & 0 \\ \infty & 0 & 0\end{array}\right), & C_{(4: 6,4: 6)}^{2}=\left(\begin{array}{ccc}0 & 0 & \infty \\ 1 & 7 & 0 \\ \infty & 0 & 0\end{array}\right), C_{(7: 9,7: 9)}^{2}=\left(\begin{array}{ccc}0 & 0 & \infty \\ 15 & 6 & 0 \\ \infty & 19 & 0\end{array}\right),\end{array}$ and let all remaining cost coefficients be set to infinity. This problem decomposes into three $(3 \times 3)$-subproblems denoted by $S_{1}, S_{2}$ and $S_{3}$, where each subproblem $S_{i}$ has three solutions $G_{1}, G_{2}$ and $G_{3}$ that have finite costs in both objectives. These three solutions have the same structure for all three subproblems and are depicted in Figure 7. Note that the cost vector of each solution $G_{j}$ of subproblem $S_{i}$ is chosen such that it corresponds to the cost vector of the path connecting node $s_{i}$ with node $s_{i+1}$ via node $s_{i j}$ in Figure 2. Consequently, there is a one-to-one correspondence between the efficient solutions of this instance of the bicriteria linear assignment problem and the efficient solutions of the bicriteria shortest path problem shown in Figure 3.

From Figure 7 it can be seen that the pairwise union of two subgraphs $G_{i}$ and $G_{j}, i \neq j$, contains exactly one cycle. According to Definition 3.4, two efficient assignments of the overall problem are thus adjacent if they differ in exactly one subproblem $S_{i}, i \in\{1,2,3\}$. Since the efficient path $P_{8}$ in Figure 3 differs from all other efficient paths in at least two connections, the corresponding assignment (consisting of $G_{2}$ in all three subproblems) differs from all other efficient assignments in at least two subproblems and is thus not adjacent to any other efficient assignment of the overall problem. 


\subsection{Transportation and transshipment problem}

Since this problem can be interpreted as a special case of the linear assignment problem (see, for example, Ehrgott and Gandibleux, 2000), the non-connectedness result of Section 3.9 can be transferred.

\subsection{The traveling salesman problem}

Paquete et al. (2004) and Paquete and Stützle (2006) introduced a combinatorial definition of adjacency for the MTSP: Two feasible tours of the MTSP are called adjacent if they differ in exactly four edges. Note that this definition corresponds to the 2-edge-exchange neighborhood of the TSP. However, the 2-edge-exchange neighborhood does not induce a canonical definition of adjacency.

Proposition 3.12 The set of optimal solutions of the single objective TSP is in general non-connected with respect to the 2-edge-exchange neighborhood.

Proof: Consider the following instance of a symmetric TSP with five nodes and distance matrix $D$ given by

$$
D=\left(\begin{array}{ccccc}
0 & 7 & 10 & 3 & 1001 \\
7 & 0 & 3 & 10 & 1000 \\
10 & 3 & 0 & 7 & 1000 \\
3 & 10 & 7 & 0 & 1001 \\
1001 & 1000 & 1000 & 1001 & 0
\end{array}\right)
$$

The problem has two optimal tours $T_{1}=(1,4,3,2,5,1)$ and $T_{2}=(1,4,5,3,2,1)$ with cost $c\left(T_{1}\right)=c\left(T_{2}\right)=2014$. However, $T_{1}$ differs from $T_{2}$ in six edges, i.e., $T_{1}$ is only contained in the 3-edge-exchange neighborhood of $T_{2}$ and not in its 2-edge-exchange neighborhood.

Note that the formulation of an alternative, MILP-based definition of adjacency is not immediate in the case of the bicriteria TSP as long as no appropriate MILP formulation of the problem is available. Moreover, it is $\mathcal{N} \mathcal{P}$-hard to decide whether two given vertices of the TSP-polytope are adjacent (Papadimitriou, 1978).

\section{Numerical Results}

All results in Section 3 are obtained from a worst-case analysis. Therefore, it is an interesting question how frequently the phenomenon "non-connected adjacency graph" occurs 
in practice. To learn more about the practical relevance of adjacency considerations, we exemplarily conduct numerical studies for the biobjective $\{0,1\}$-knapsack problem with bounded cardinality and the biobjective $\{0,1\}$-multiple choice knapsack problem (cf. Section 3.5). All in all, more than six million randomly generated problem instances have been analyzed.

Before describing the design of the numerical experiments in detail, one has to be aware of some facts being characteristic for our problem. First, it is not sufficient to compute just one efficient solution for each nondominated point. All efficient solutions have to be found. Consequently, an algorithm enumerating all alternative solutions for the same nondominated outcome is required. Second, after having found all efficient solutions, their adjacency relationships have to be explored. The set of efficient solutions has to be traversed several times, solutions have to be compared pairwise, and clusters of efficient solutions have to be built. This post-solution analysis requires a substantial amount of computation time. Third, non-connected adjacency graphs cannot be expected to occur with a high frequency in randomly generated problem instances. Conclusions can therefore not be drawn on the basis of just a few dozen instances. For each set-up thousands of instances have to be generated and tested to yield representative results. Fourth, computation power as well as computation time are limited.

Recapitulating, one can conclude that under these circumstances the instances treated in our study have to be rather small and do not nearly match the sizes of state-of-the-art benchmark problems.

\subsection{Biobjective $\{0,1\}$-knapsack problems with bounded cardinality}

For the computation of the efficient and nondominated set of the generated instances of the biobjective $\{0,1\}$-knapsack problem with bounded cardinality $K P(n, k)$ we used a dynamic programming approach for general multiple objective knapsack problems developed in Klamroth and Wiecek (2000). We implemented Model III for the binary case (cf. the original work of Klamroth and Wiecek, 2000). Using this approach, we can solve $k$ knapsack instances $K P(n, 1), \ldots, K P(n, k)$ simultaneously, i.e., without any additional computational cost. For each efficient solution of one of the problem instances, we store a binary vector representing this solution in a list. Recall from Section 3.5 that two efficient solutions are defined to be adjacent if their Hamming distance is equal to two. Starting with the first efficient solution in the list, we find all its neighbors (by pairwise computations of Hamming distances with all the remaining solutions) in the list and mark them with a certain label. Different labels signalize different adjacency clusters. We proceed likewise with the second solution in the list. Eventually, adjacency clusters have to be 


\begin{tabular}{|c|c|c|c|c|c|c|c|}
\hline Method / Setup & $10 / 9 / 10$ & $20 / 10 / 10$ & $30 / 15 / 10$ & $40 / 20 / 10$ & $60 / 30 / 10$ & $80 / 40 / 20$ & $100 / 50 / 20$ \\
\hline 1 & 50000 & 20000 & 1000 & 1000 & - & - & - \\
\hline 2 & 50000 & 20000 & 1000 & 1000 & - & - & - \\
\hline 3 & 50000 & 20000 & 1000 & 1000 & - & - & - \\
\hline 4 & 50000 & 20000 & 1000 & 1000 & - & - & - \\
\hline 5 & 50000 & 20000 & 1000 & 1000 & - & - & - \\
\hline 6 & 50000 & 50000 & 50000 & 10000 & 10000 & 10000 & 1000 \\
\hline
\end{tabular}

Table 2: Setup of computational experiments for the biobjective $\{0,1\}$-knapsack problem with bounded cardinality

\begin{tabular}{|c|c|c|c|c|c|c|c|}
\hline Method / Setup & $10 / 9 / 10$ & $20 / 10 / 10$ & $30 / 15 / 10$ & $40 / 20 / 10$ & $60 / 30 / 10$ & $80 / 40 / 20$ & $100 / 50 / 20$ \\
\hline 1 & 0 & 0 & 0 & 2 & - & - & - \\
\hline 2 & 0 & 2 & 1 & 1 & - & - & - \\
\hline 3 & 0 & 2 & 0 & 0 & - & - & - \\
\hline 4 & 0 & 1 & 0 & 0 & - & - & - \\
\hline 5 & 0 & 1 & 1 & 0 & - & - & - \\
\hline 6 & 0 & 0 & 0 & 0 & 0 & 1 & 0 \\
\hline
\end{tabular}

Table 3: Number of instances with adjacency graph having more than one connected component

merged, i.e., markers have to be re-assigned. After having processed all efficient solutions, the number of different markers indicate the number of clusters.

\section{Purpose and setup of the numerical study}

The aim of the numerical study is to report the number of adjacency clusters of randomly generated instances of $K P(n, k)$ when

a) $n$ increases,

b) $k$ increases for fixed $n$, and

c) the objective coefficients are generated according to different methods.

We generated seven problem setups and for each setup we used six different methods to generate the objective coefficients. In the first row of Table 2, we use a scheme of the form Pos $1 /$ Pos $2 /$ Pos 3 to code these seven setups. Pos 1 specifies the total number $n$ of items. The upper bound $k$ for the right hand side parameter of the knapsack constraint is specified under Pos2. We determined the adjacency graph for all possible right hand sides $i \in\{1, \ldots, \operatorname{Pos} 2\}$. Finally, the coefficients of the first objective $c^{1}$ were chosen in the interval $[0, r]$, where $r=\operatorname{Pos} 1 \cdot \operatorname{Pos} 3$. The coefficients of the second objective $c^{2}$ were chosen according to six different methods which were motivated by the study of Pedersen et al. (2005) and which are described in the following. 
Method 1: $c^{1}$ was sorted in decreasing, $c^{2}$ in increasing order to obtain pairwise nondominated profit vectors. Weakly-dominated vectors were omitted.

Method 2: The profit vectors $p_{1}:=\left(c_{1}^{1}, c_{1}^{2}\right)^{T}=(r, 0)^{T}$ and $p_{n}:=\left(c_{n}^{1}, c_{n}^{2}\right)^{T}=(0, r)^{T}$ were fixed at the beginning. The remaining vectors were chosen within the triangle $(0,0)^{T}, p_{1}$ and $p_{n}$. Preferably pairwise non-dominated profit vectors were generated. Only profit matrices with a few dominated profit vectors were accepted.

Method 3: The profit vectors were generated as in Method 2, but now within the triangle $(r, r)^{T}, p_{1}$ and $p_{n}$.

Method 4: The profit vectors $p_{1}$ and $p_{n}$ were fixed like in Method 2. The remaining vectors were generated spread around the concave part of the half circle with midpoint $(0,0)^{T}$ connecting the points $p_{1}$ and $p_{n}$. Preferably pairwise non-dominated profit vectors were generated and profit matrices with only a few dominated profit vectors were accepted.

Method 5: The profit vectors were generated as in Method 4, but now spread around the convex half circle with midpoint $(r, r)^{T}$ connecting $p_{1}$ and $p_{n}$.

Method 6: The entries of the profit matrix were generated uniformly at random.

The entries of Table 2 correspond to the number of instances which were processed for each of the setups. Note that these numbers are not always the same. Some setups result in more difficult instances and thus, we could only process fewer instances in a reasonable amount of time. A dash indicates that this setup was not tested due to its numerical difficulty.

\section{Interpretation of the numerical results}

Table 3 presents the number of non-connected adjacency graphs that were found for each setup. For the generated instances, only very few adjacency graphs are non-connected. Nevertheless, we found for each of the six data generation methods at least one instance possessing a non-connected adjacency graph. Based on the small number of components, there do not seem to exist significant trends - neither with respect to increasing $k$ or $n$ nor with respect to some particular generation method for the data. For the case of randomly generated profit matrices (Method 6), non-connected adjacency graphs seem to occur extremely rarely. As mentioned in da Silva et al. (2004), an item $x_{i}$ corresponding to a dominated profit vector $p_{i}$ can only be contained in an efficient knapsack if at least one of the items $x_{j}$ corresponding to profit vectors $p_{j}$ dominating $p_{i}$ is also contained in 
the knapsack. The set of all efficient solutions of such a problem consists of a few number of elements and is more structured than in the case when only pairwise non-dominated profit vectors are considered. For the problem size (40/20/10), the maximum number of elements of an efficient set for a problem instance generated by Method 6 is given by 290 while for the other methods the maximum number does not fall below 1392 and has a maximum value of over 5300 elements for a problem instance generated by Method 2 . Unfortunately, Method 6 seems to be the "standard" way to generate data when testing an algorithm numerically. Yet, for algorithms based on neighborhood search, this problem class seems to be quite uninteresting.

\subsection{Biobjective $\{0,1\}$-multiple choice knapsack problems}

The second part of the numerical study is devoted to the biobjective $\{0,1\}$-multiple choice knapsack problem introduced in Section 3.5. Recall that this problem is closely related to the biobjective $\{0,1\}$-knapsack problem with bounded cardinality. Yet, this problem behaves quite differently with respect to the adjacency issue.

Suppose that a biobjective $\{0,1\}$-multiple choice knapsack problem is given with $n$ baskets and $k$ possible items per basket. To obtain the set of efficient solutions $X_{E}$, we use a simple dynamic programming scheme. In the $i$-th step, $i=1, \ldots, n$, we combine every solution being efficient for the problem with baskets $B_{1}, \ldots, B_{i-1}$, with the items in basket $i$. Dominated solutions are deleted. The remaining solutions form the set of efficient solutions for the problem with baskets $B_{1}, \ldots, B_{i-1}$. Note that the items in each basket should be pairwise nondominated since dominated items are never included in an efficient solution. It should be pointed out that applying this scheme, we solve in fact not only the problem for $n$ baskets, but $n$ different problems for $i, i=1, \ldots, n$, baskets. Similar to the previous study, a post-optimality procedure is applied to $X_{E}$ to retrieve the adjacency information.

\section{Purpose and setup of the numerical study}

We study the frequency of problems with non-connected adjacency graphs when

a) the number of baskets increases from 1 to $n$, and

b) the (fixed) number of items per basket increases.

As in Section 4.1, we use a scheme Pos1/Pos2/Pos3 coding the setup of the instances. Pos1 stands for the number of baskets while the number of items per basket is given in Pos2. The integer cost coefficients are taken from the interval $[1$, Pos $1 \cdot$ Pos 3$]$ according 


\begin{tabular}{|c|c|c|c|}
\hline Setup of test instances & $20 / 5 / 10$ & $20 / 10 / 10$ & $20 / 15 / 10$ \\
\hline Number of instances generated & 10000 & 5000 & 1000 \\
\hline Number of instances having a non-connected adjacency graph & 118 & 295 & 111 \\
\hline
\end{tabular}

Table 4: Setup of computational experiments for the biobjective $\{0,1\}$-multiple choice knapsack problem with uniform weights

\begin{tabular}{|c|c|c|c|}
\hline Setup of test instances & $20 / 5 / 10$ & $20 / 10 / 10$ & $20 / 15 / 10$ \\
\hline Instances with one cluster & 199882 & 99705 & 19889 \\
\hline Instances with two clusters & 115 & 282 & 100 \\
\hline Instances with three clusters & 3 & 13 & 11 \\
\hline Maximal distance between two clusters & 2 & 2 & 2 \\
\hline Maximal distance between three clusters & 3 & 4 & 8 \\
\hline
\end{tabular}

Table 5: Number of connected components (clusters) in the adjacency graph and maximal distance between two components for the biobjective $\{0,1\}$-multiple choice knapsack problem

to Method 1 in Section 4.1. Table 4 reports the setups and the number of instances we have tested.

\section{Interpretation of the numerical results}

For each of the setups, Table 4 contains the number of instances possessing a non-connected adjacency graph cumulated over all $i=1, \ldots, 20$ baskets. One obvious difference to the results obtained in Section 4.1 is that non-connected adjacency graphs occur far more often for multiple choice knapsack problems. Figure 8 shows the (normalized) number of instances with non-connected adjacency graph per thousand instances tested. Detailed results for each problem with $i$ baskets, $i=1, \ldots, 20$, are reported. The dotted line, the dashed line, and the solid line correspond to the setups with 5, 10, and 15 items per basket, respectively. All curves are (slightly) increasing, i.e., "non-connectedness" happens more often with an increasing number of baskets. Furthermore, the more items per basket, the higher the likelihood for having a non-connected adjacency graph. Table 5 provides more details about the character of the clusters. Among those instances with non-connected adjacency graph, two clusters appear more often than three clusters. However, with increasing number of items per basket three clusters are getting more likely. Interestingly, the maximal distance between clusters of instances with two clusters only is never greater than 2. The maximal (pairwise) distance between three clusters, however, can be as much as 8 . 


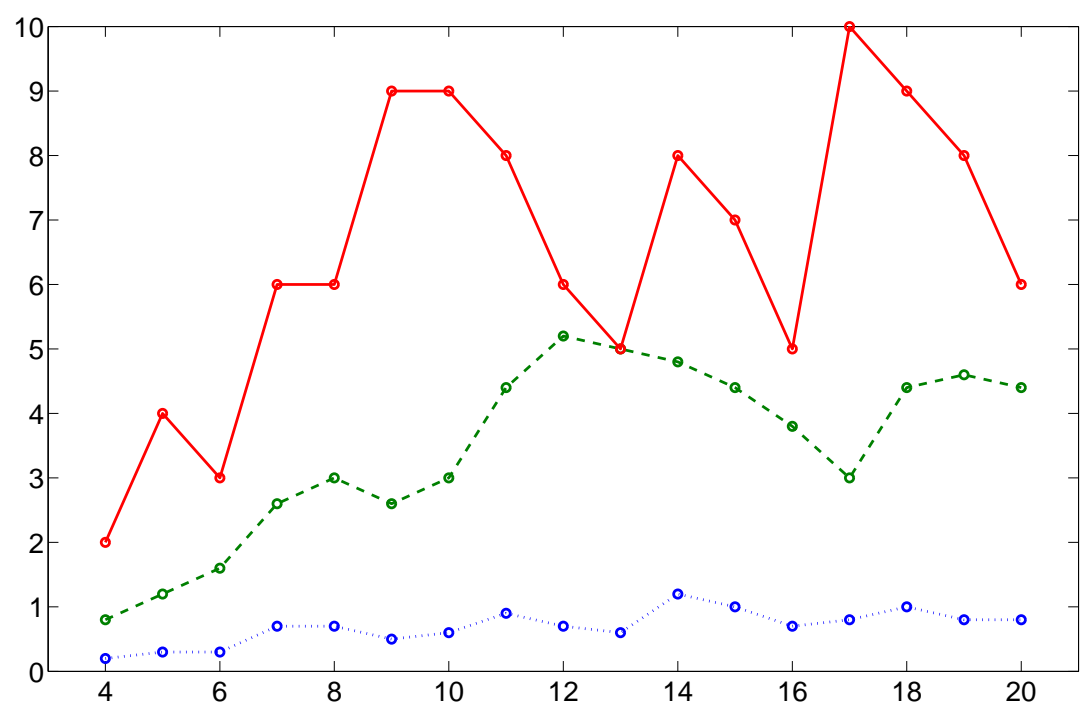

Figure 8: Number of instances per thousand ( $y$-axis) with non-connected adjacency graph for $i=1, \ldots, 20$ baskets ( $x$-axis) for 5 (dotted line), 10 (dashed line), and 15 (solid line) items per basket

\section{Concluding remarks}

To summarize the discussion above, the two problems treated in this section behave very differently with respect to adjacency although their combinatorial structure seems quite similar. This shows how careful one has to be with statements about adjacency and it also shows the limitations of our study: No results about larger instances, about problems with more than two objective functions, and about other problems are available so far.

\section{Conclusion and current research directions}

As in the case of single objective combinatorial optimization, the question of adjacency of solutions is one of the core aspects in multiple objective combinatorial optimization (MOCO). The concept of adjacency of optimal solutions in multiple objective problems certainly exceeds its single criterion analogon in terms of complexity because of a more involved optimality concept. Maybe it is due to this increased complexity that research on this subject has widely been neglected. To the best of our knowledge there does not exist (correct) exact algorithms for computing the set of efficient solutions based on neighborhood structures, nor does the literature formalize different notions of adjacency. Beyond, adjacency of MOCO problems has not been investigated numerically. 
The aim of our work is threefold. First, we formally introduce two different concepts of adjacency. One class of adjacency concepts relies on problem-dependent combinatorial structures, while the other one is based on appropriate models for the problem and, ultimately, goes back to the definition of adjacency for multiple objective linear programs. Second, we survey the current state of the art and supplement it with our own findings. As a result, we list eleven combinatorial optimization problems and discuss their adjacency properties. Third, we conduct numerical experiments to analyze the adjacency structure of two special types of bi-objective knapsack problems. Although being structurally related and possessing a non-connected adjacency graph in general, these knapsack problems differ significantly in the practical occurrence of adjacency.

Our work should be understood as a first step towards an in-depth investigation of adjacency in MOCO problems. In our opinion, several research directions seem to be promising and are currently under investigation:

- Although we prove the non-connectedness of many fundamental MOCO problems in general, special variants of these problems might possess a connected adjacency graph (cf. da Silva et al., 2004). Further explorations of the structure, the size and the geometry of connected components and their dependencies on the choice of the problem data are currently the main focus of our work.

- The structural results presented in Section 3 are based on a worst-case analysis. Studying theoretically the average case gives detailed information about the expected occurrence of adjacency in practical problems and might justify the application of adjacency-based algorithms even for problems having non-connected adjacency graphs in general.

- If an average-case analysis is not possible for some problems, intensive numerical investigations about the adjacency behavior might provide empirical evidence for the effectiveness of adjacency-based algorithms.

- Another interesting stream of research is the development of new definitions of adjacency possibly yielding connected adjacency graph for a wider class of MOCO problems.

\section{References}

Balinski, M. L. and A. Russakoff (1974). On the assignment polytope. SIAM Review 16, $516-525$. 
da Silva, C. G., J. Clímaco, and J. Figueira (2004). Geometrical configuration of the Pareto frontier of bi-criteria $\{0,1\}$-knapsack problems. Working paper 16-2004, Institute for Systems and Computers Engineering, Coimbra, Portugal.

Ehrgott, M. (2005). Multicriteria Optimization. Berlin, Heidelberg: Springer.

Ehrgott, M. and X. Gandibleux (2000). A survey and annotated bibliography of multiobjective combinatorial optimization. OR Spektrum 22, 425-460.

Ehrgott, M. and K. Klamroth (1997). Connectedness of efficient solutions in multiple criteria combinatorial optimization. European Journal of Operational Research 97, 159166.

Geist, D. and E. Y. Rodin (1992). Adjacency of the 0-1 knapsack problem. Computers and Operations Research 19, 797-800.

Gorski, J. (2004). Analysis of the connectedness of Pareto-optimal solutions in multiple criteria combinatorial optimization. Master's thesis, University of Erlangen-Nuremberg, Germany. In German.

Hausmann, D. (1980). Adjacency on Polytopes in Combinatorial Optimization. Königstein/Ts.: Verlag Anton Hain.

Helbig, S. (1990). On the connectedness of the set of weakly efficient points of a vector optimization problem in locally convex spaces. Journal of Optimization Theory and Applications 65, 257-271.

Isermann, H. (1977). The enumeration of the set of all efficient solutions for a linear multiple objective program. Operations Research Quarterly 28, 711-725.

Klamroth, K. and M. Wiecek (2000). Dynamic programming approaches to the multiple criteria knapsack problem. Naval Research Logistics 47, 57-76.

Martins, E. (1984). On a multicriteria shortest path problem. European Journal of Operational Research 16, 236-245.

Naccache, P. (1978). Connectedness of the set of nondominated outcomes. Journal of Optimization Theory and Applications 25, 459-467.

Nemhauser, G. and L. Wolsey (1999). Integer and Combinatorial Optimization. New York: John Wiley \& Sons, Inc. 
O'Sullivan, M. and C. Walker (2004). Connecting efficient knapsacks - experiments with the equally-weighted bi-criteria knapsack problem. In 39th Annual ORSNZ Conference, University of Auckland, Auckland, New Zealand.

Papadimitriou, C. (1978). The adjacency relation on the traveling salesman polytope is NP-complete. Mathematical Programming 14, 312-324.

Paquete, L., M. Chiarandini, and T. Stützle (2004). Pareto local optimum sets in the biobjective traveling salesman problem: An experimental study. In X. Gandibleux, M. Sevaux, K. Srensen, and V. T'kindt (Eds.), Metaheuristics for Multiobjective Optimisation, Volume 535 of Lecture Notes in Economics and Mathematical Systems. Springer Verlag.

Paquete, L. and T. Stützle (2006). Clusters of non-dominated solutions in multiobjective combinatorial optimization. In MOPGP'06: 7th International Conference on MultiObjective Programming and Goal Programming, Loire valley, Tours.

Pedersen, C. (2006). Multicriteria Discrete Optimization - and Related Topics. Ph. D. thesis, University of Aarhus.

Pedersen, C., L. Nielsen, and K. Andersen (2005). On the bicriterion multi modal assignment problem. Working paper WP-2005-3, Department of Operations Research, University of Aarhus.

Przybylski, A., X. Gandibleux, and M. Ehrgott (2006). The biobjective integer minimum cost flow problem - incorrectness of Sedeño-Noda and González-Martín's algorithm. Computers and Operations Research 33(5), 1459-1463.

Sedeño-Noda, A. and C. González-Martín (2001). An algorithm for the biobjective integer minimum cost flow problem. Computers and Operations Research 28, 139-156.

Warburton, A. (1983). Quasiconcave vector maximization: Connectedness of the sets of Pareto-optimal and weak Pareto-optimal alternatives. Journal of Optimization Theory and Applications 40, 537-557. 